\title{
On the derived tensor product functors for (DF)- and Fréchet spaces
}

\author{
by \\ OĞUZ VAROL (Wuppertal)
}

\begin{abstract}
For a (DF)-space $E$ and a tensor norm $\alpha$ we investigate the derivatives $\operatorname{Tor}_{\alpha}^{l}(E, \cdot)$ of the tensor product functor $E \widetilde{\otimes}_{\alpha} \cdot: \mathcal{F S} \rightarrow \mathcal{L S}$ from the category of Fréchet spaces to the category of linear spaces. Necessary and sufficient conditions for the vanishing of $\operatorname{Tor}_{\alpha}^{1}(E, F)$, which is strongly related to the exactness of tensored sequences, are presented and characterizations in the nuclear and (co-)echelon cases are given.
\end{abstract}

Introduction. Locally convex tensor products go back to A. Grothendieck, who studied them in detail in the 1950's ([5]). Their tremendous importance is, inter alia, due to the fact that in many cases, spaces of operators, vector-valued functions etc. can be represented as tensor products. In this language, surjectivity problems that commonly appear e.g. for vectorvalued partial differential operators with constant coefficients, correspond to exactness properties of tensored sequences (see [12], [29] and [25]).

In the early 1970's V. P. Palamodov laid the basis for the usage of categorical and homological methods in functional analysis ([19]). Unfortunately, tensor product functors and their derivatives were not mentioned in that fundamental paper. While Ext ${ }^{1}$ for Fréchet spaces, whose vanishing is equivalent to the splitting of so-called extensions, has been successfully investigated since, first of all by D. Vogt $([26,28])$, the powerful apparatus of homological algebra was completely avoided in studying the exactness of tensored sequences.

This article is devoted to derivatives of tensor product functors from the category of Fréchet spaces to the category of linear spaces, whose vanishing is close to the exactness of tensored sequences.

In the first section we look at this problem by decomposing it into its components: tensoring and completing. In this context one has to emphasize

2000 Mathematics Subject Classification: Primary 46M18, 46A32; Secondary 46A04, 46A11, 46A45.

Key words and phrases: derived tensor product functors, topological tensor products, projective limits, Fréchet spaces, (DF)-spaces. 
conditions which depend on the exact sequence considered and the space which is being tensored. The first approach leads directly to the so-called $\otimes$-sequences of W. Kaballo and D. Vogt ([12]), while the second to $\pi$ - and $\varepsilon$-spaces as defined by R. Hollstein ([7-9]).

In the second section we will formulate and prove one of the central theorems of this paper. It states that in most applications, there is a close relation between the derivatives of so-called $\sigma$-stable functors, i.e., functors that commute with the differentials of the proj-functor, and the $\operatorname{proj}^{1}$-functor. The vanishing of $\operatorname{proj}^{1}$ was studied by many authors; in the first place one has to mention V. P. Palamodov ([19]) and V. S. Retakh.

The third section gives first examples for derivatives of tensor product functors and is followed by a technical section where we use well-known methods of proj ${ }^{1}$-theory (see [1] resp. [4] and [13]) to deduce necessary and sufficient conditions for the vanishing of Tor ${ }^{1}$. Here we will concentrate on the important case where the tensored space is an (LB)-space. Interchangeability properties of (LB)-spaces with Banach spaces in the tensorial sense turn out to be very fruitful for computations.

In [24] the author generalized and extended a classical and deep result of A. Grothendieck, who characterized topological properties of the projective tensor product of a coechelon space and an echelon space of type one ([5, II, $\S 4$, no. 3, Theorem 1]). In the last section we shall formulate four standard cases for Tor-theory, in analogy to the four standard cases in Ext-theory ([26], [4]), and give characterizations for the vanishing of Tor $^{1}$ in all of them, i.e., in nuclear and (co-)echelon cases.

1. Some basics from category theory and tensor products. We expect the reader to be familiar with the concept of categories, functors and natural transformations. Unless otherwise stated, we assume functors to be covariant and locally convex spaces to be separated. $B_{X}$ will denote the closed unit ball of a normed space $X$. For our general notation of locally convex spaces and tensor products we refer to [17] and [10].

As mentioned in the introduction we wish to study the exactness of the continuation of tensored sequences to their completion. For this purpose we first repeat some of the needed categorical terms (see [19] and [31] for details), and apply these in a first rudimentary step to our situation.

Definition 1.1. An additive category $\mathcal{C}$ is called semi-abelian if every $\mathcal{C}$-morphism $f$ has a kernel and a cokernel and the induced $\mathcal{C}$-morphism $\bar{f}: \operatorname{coim}(f) \rightarrow \operatorname{im}(f)$ is a bimorphism, i.e., a monomorphism $(\operatorname{ker}(\bar{f})=0)$ and an epimorphism $(\operatorname{coker}(\bar{f})=0)$. If in addition every $\mathcal{C}$-morphism $f$ is a homomorphism, i.e., $\bar{f}$ is an isomorphism, then $\mathcal{C}$ is called abelian. We say 
that a $\mathcal{C}$-homomorphism is a monohomomorphism (epihomomorphism) if it is a monomorphism (epimorphism, respectively).

We will deal primarily with the following examples: the category $\mathcal{L S}$ of linear spaces and linear maps is abelian, whereas the category $\mathcal{L C S}$ of locally convex spaces, which are not necessarily separated, and continuous linear maps is semi-abelian. The category $\mathcal{F} \mathcal{S}$ of Fréchet spaces and continuous linear maps is also semi-abelian. Monohomomorphisms (epihomomorphisms) in $\mathcal{L C S}$ and $\mathcal{F S}$ are nothing else than topological embeddings (surjective homomorphisms, respectively).

Now let $\mathcal{C}$ be a semi-abelian category. We say that a $\mathcal{C}$-sequence

$$
\cdots \rightarrow A^{n-1} \stackrel{d^{n-1}}{\rightarrow} A^{n} \stackrel{d^{n}}{\rightarrow} A^{n+1} \rightarrow \cdots
$$

is exact at $A^{n}$ if $d^{n-1}$ and $d^{n}$ are homomorphisms and $\operatorname{im}\left(d^{n-1}\right)=\operatorname{ker}\left(d^{n}\right)$. Furthermore we say that the sequence is exact if it is everywhere exact.

Definition 1.2. An additive functor $R: \mathcal{C} \rightarrow \mathcal{D}$ between semi-abelian categories is called

(i) left exact if for all exact $\mathcal{C}$-sequences $0 \rightarrow F \rightarrow G \rightarrow H$ the $\mathcal{D}$-sequence $0 \rightarrow R(F) \rightarrow R(G) \rightarrow R(H)$ is exact,

(ii) exact if for all exact $\mathcal{C}$-sequences $0 \rightarrow F \rightarrow G \rightarrow H \rightarrow 0$ the $\mathcal{D}$-sequence $0 \rightarrow R(F) \rightarrow R(G) \rightarrow R(H) \rightarrow 0$ is exact.

EXAMPLE 1.3.

(i) It is well-known that projective and inductive spectra and their limits play an important role in the structure theory of Fréchetand (DF)-spaces and in applications of Banach space theory to these spaces. On the other hand, it will turn out that the projective limit functor, or to be more precise, its first derivative, is one of our main objects of study. A straightforward calculation shows that the projective limit functor proj : $\mathcal{L S}^{\mathbb{N}} \rightarrow \mathcal{L S}$,

$$
\begin{aligned}
& \mathcal{F}=\left(F_{n}, \varrho_{n+1}^{n}\right) \mapsto\left\{x \in \prod_{n=1}^{\infty} F_{n}: \varrho_{n+1}^{n}\left(x_{n+1}\right)=x_{n} \text { for all } n\right\}, \\
& \left(f_{n}\right)_{n} \mapsto\left(x \mapsto\left(f_{n}(x)\right)_{n}\right)
\end{aligned}
$$

on the category of (countable) projective spectra of linear spaces is left exact.

(ii) For a linear space $E$ the tensor product functor $E \otimes \cdot: \mathcal{L S} \rightarrow \mathcal{L S}$, $F \mapsto E \otimes F, f \mapsto \operatorname{id}_{E} \otimes f$, is exact (see for instance [10, Proposition 1.6 .3 and its proof]).

(iii) The completion functor ${ }^{\sim}: \mathcal{L C S} \rightarrow \mathcal{L C S}, F \mapsto \widetilde{F}, f \mapsto \widetilde{f}$, is left exact, where $\widetilde{F}$ denotes the Hausdorff completion of $F$ (see [19, Proposition 10.3] for a nice homological proof). 
In connection with [19, Proposition 4.2] we obtain Then

Corollary 1.4. Let $f: F \rightarrow G$ and $g: G \rightarrow H$ be $\mathcal{L} \mathcal{C} \mathcal{S}$-morphisms.

(a) If $g$ is a homomorphism, then $\operatorname{ker}(\widetilde{g})=\widetilde{\operatorname{ker}(g)}$.

(b) If $f$ is a monohomomorphism, then $\operatorname{im}(\tilde{f})=\widetilde{\operatorname{im}(f)}$.

(c) The morphism $g$ is a (mono-)homomorphism if and only if $\widetilde{g}$ is a (mono-)homomorphism.

If $E=\operatorname{ind}_{N} E_{N}$ is a non-complete (LB)-space (these are always assumed to be injective), the completion of the canonical epihomomorphism $g: \bigoplus_{N} E_{N} \rightarrow E,\left(x_{N}\right)_{N} \mapsto \sum x_{N}$, is not surjective and therefore not an epihomomorphism. However, in the metrizable case the behaviour of epihomomorphisms is good: Using [17, Lemma 3.9] it suffices to show that the completed map is nearly open, i.e., the closure of the image of a neighbourhood of zero is again a neighbourhood of zero, which is immediate. Consequently, due to Example 1.3(iii) the completion of an exact sequence of metrizable locally convex spaces is again topologically exact.

For a monomorphism $f: F \rightarrow G$ between locally convex spaces the map $\tilde{f}$ in general does not remain a monomorphism:

EXAMPLE 1.5. Let $(X,\|\cdot\|)$ be an arbitrary infinite-dimensional Banach space, $\left(y_{n}\right)_{n \in \mathbb{N}}$ a sequence of linearly independent elements in $X$ of norm one, $Y:=\operatorname{span}\left(\left\{y_{1}, y_{2}, \ldots\right\}\right)$, and $Z$ an algebraic complement of $Y$ in $X$. Then every $x \in X$ has a unique representation as $x=y+z$ with $y=\sum_{n=1}^{N} \lambda_{n} y_{n}$ $\in Y, N \in \mathbb{N}, \lambda_{n} \in \mathbb{K}$ and $z \in Z$. Setting $\||x|\|:=\sum_{n=1}^{N} n\left|\lambda_{n}\right|+\|z\|$, we get another norm on $X$, which satisfies $\|\cdot\| \leq\|\cdot \cdot\| \mid$, but which is not equivalent to $\|\cdot\|$. If $f:(X,\|\cdot\| \|) \rightarrow(X,\|\cdot\|)$ denotes the identity, an application of the open mapping theorem shows that $\tilde{f}$ is an epihomomorphism but is not injective.

We have already seen that in the purely algebraic case tensor product functors behave very well (Example 1.3(ii)). Now, the central question is: What changes if we consider locally convex spaces and endow the tensor products with natural locally convex topologies like the projective or injective tensor norm topology and pass to the completion?

Let us summarize a few results regarding this question and the projective tensor product. For the injective tensor product we refer to [10, Section 16.2] and leave the corresponding statements to the reader.

REMARK 1.6. Recall that for locally convex spaces $E$ and $F$ the projective tensor product topology $\pi$ is the finest locally convex topology on $E \otimes F$ such that the canonical map $E \times F \rightarrow E \otimes F$ is continuous. This space is denoted by $E \otimes_{\pi} F$ and its completion by $E \widetilde{\otimes}_{\pi} F$. Using Corollary 1.4(a), 
Example 1.3(ii) and the fact that the projective tensor product respects epihomomorphisms ([10, Proposition 15.2.1]) we conclude: Let $E$ be a locally convex space and $0 \rightarrow F \stackrel{f}{\rightarrow} G \stackrel{g}{\rightarrow} H \rightarrow 0$ an exact sequence of locally convex spaces. Then in the sequence

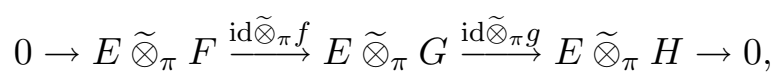

$\operatorname{im}\left(\operatorname{id}_{E} \widetilde{\otimes}_{\pi} f\right)$ is dense in $\operatorname{ker}\left(\operatorname{id}_{E} \widetilde{\otimes}_{\pi} g\right)$ and $\operatorname{id}_{E} \widetilde{\otimes}_{\pi} g$ is a homomorphism.

It is well-known that in general $\operatorname{id}_{E} \widetilde{\otimes}_{\pi} f$ is not a monohomomorphism ([10, Example 15.2.2]). The following example shows that $\operatorname{id}_{E} \widetilde{\otimes}_{\pi} f$ is not injective in general. Suppose $E$ is a Banach space without the approximation property (a.p.), i.e., there is a Banach space $F$ such that the map $i_{E, F}$ : $E \widetilde{\otimes}_{\pi} F \rightarrow E \widetilde{\otimes}_{\varepsilon} F$ is not injective $([2,5.6])$. Consider the monohomomorphism $f: F \rightarrow G:=l_{\infty}\left(B_{F}^{\circ}\right), x \mapsto\left(x^{\prime} \mapsto x^{\prime}(x)\right)$, from $F$ into the space of bounded maps on the $\sigma\left(F^{\prime}, F\right)$-compact set $B_{F}^{\circ}$. As $G$ has the a.p. the map $i_{E, G}$ is injective and the assertion follows from the commutativity of the diagram

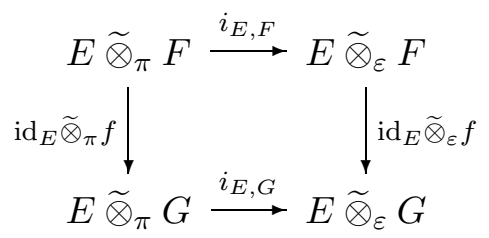

Furthermore the $\operatorname{map} \operatorname{id}_{E} \widetilde{\otimes}_{\pi} g$ has dense range, but is in general not surjective: Consider a nuclear, non-splitting sequence of complete locally convex spaces, for instance the Borel sequence $0 \rightarrow J_{\{0\}} \rightarrow \mathcal{E}([-1,1]) \stackrel{g}{\rightarrow} \omega \rightarrow 0$, where $g(f):=\left(f^{(n)}(0)\right)_{n \in \mathbb{N}_{0}}$ (cf. [17, Aufgabe 1, §30]). The map $\operatorname{id}_{H^{\prime}} \widetilde{\otimes}_{\pi} g$ : $H^{\prime} \widetilde{\otimes}_{\pi} G=L(H, G) \rightarrow L(H, H)=H^{\prime} \widetilde{\otimes}_{\pi} H$ is not surjective, as a lifting of $\operatorname{id}_{H}$ would be a right inverse of $g$.

At first sight there are now two obvious ways to achieve more exactness. The first one is to make further assumptions on the exact sequence considered. This leads to the so-called $\otimes$-sequences of W. Kaballo and D. Vogt: an exact sequence $0 \rightarrow F \stackrel{f}{\rightarrow} G \stackrel{g}{\rightarrow} H \rightarrow 0$ of locally convex spaces is called a $\otimes$-sequence if $\operatorname{id}_{E} \otimes_{\pi} f$ remains a monohomomorphism (or equivalently, if $\operatorname{id}_{E} \otimes_{\varepsilon} g$ remains an epihomomorphism) for all locally convex spaces (or equivalently for all Banach spaces) $E$. For instance, exact sequences of nuclear spaces are $\otimes$-sequences. For further equivalent conditions and examples we refer to [12]. In this terminology [12, Satz 1.5] becomes

Corollary 1.7.

(a) Let $0 \rightarrow F \rightarrow G \rightarrow H \rightarrow 0$ be $a \otimes$-sequence. Then the sequences $0 \rightarrow$ $E \widetilde{\otimes}_{\pi} F \rightarrow E \widetilde{\otimes}_{\pi} G \rightarrow E \widetilde{\otimes}_{\pi} H$ and $0 \rightarrow E \widetilde{\otimes}_{\varepsilon} F \rightarrow E \widetilde{\otimes}_{\varepsilon} G \rightarrow E \widetilde{\otimes}_{\varepsilon} H$ are topologically exact for all locally convex spaces $E$. 
(b) If $0 \rightarrow F \rightarrow G \rightarrow H \rightarrow 0$ is an exact sequence of Fréchet spaces, then the following conditions are equivalent:

(i) $0 \rightarrow F \rightarrow G \rightarrow H \rightarrow 0$ is a $\otimes$-sequence.

(ii) The $E \widetilde{\otimes}_{\pi} \cdot$-tensored sequence is exact for all Fréchet spaces (Banach spaces) E.

(iii) The $E \widetilde{\otimes}_{\varepsilon} \cdot-$ tensored sequence is exact for all Fréchet spaces (Banach spaces) E.

Proof. (a) This follows from Example 1.3(ii), the fact that the projective (resp. injective) tensor product respects epihomomorphisms (resp. monohomomorphisms), and Example 1.3(iii).

(b) The implications (i) $\Rightarrow$ (ii) and (i) $\Rightarrow$ (iii) are immediate from (a) and the remarks after Corollary 1.4; the others follow from Corollary 1.4(c) and Example 1.3(ii).

The second method to guarantee more exactness is to make assumptions on the space $E$. A locally convex space $E$ is called an $\varepsilon$-space (resp. a $\pi$-space) in the sense of R. Hollstein if for all epihomomorphisms $g$ (resp. monohomomorphisms $f$ ) of locally convex spaces (or equivalently, of Banach spaces) the map $\operatorname{id}_{E} \otimes_{\varepsilon} g$ (resp. $\operatorname{id}_{E} \otimes_{\pi} f$ ) is again a homomorphism ([8]). In that paper R. Hollstein also showed that the $\varepsilon$-Banach spaces (resp. $\pi$-Banach spaces) are exactly the $\mathcal{L}_{\infty}$-spaces (resp. $\mathcal{L}_{1}$-spaces) (see [14] for the notion of $\mathcal{L}_{p}$-spaces). For instance, nuclear spaces are $\varepsilon$ - and $\pi$-spaces. We will consider further examples at a later point.

Arguing as in the proof of Corollary 1.7, we obtain

COROLlary 1.8. If $E$ is an $\varepsilon$-space (resp. a $\pi$-space), then the functor $E \widetilde{\otimes}_{\varepsilon} \cdot: \mathcal{L C S} \rightarrow \mathcal{L C S}\left(\right.$ resp. $\left.E \widetilde{\otimes}_{\pi} \cdot: \mathcal{L C S} \rightarrow \mathcal{L C S}\right)$ is left exact. For a Fréchet space $E$ the converse is also true.

Though we mainly concentrate on the projective and injective tensor product we will be able to translate without any additional effort some of our results to the more general case of so-called tensor norm topologies. Based on A. Grothendieck's tensor norms, these were introduced by J. Harksen. For details on the following see [2] and [6]. Roughly speaking, a tensor norm $\alpha$ assigns to each pair of normed spaces a norm on their tensor product which is reasonable in the sense that $\varepsilon \leq \alpha \leq \pi$, and has the metric mapping property, i.e., the operator norm of tensored maps is bounded above by the product of the norms of the maps. Such a tensor norm induces in a natural way a locally convex topology on the tensor product of two given locally convex spaces by using their local Banach spaces.

2. Derived functors and their computation. As far as the author knows, it was V. P. Palamodov who introduced (co-)homological methods 
to functional analysis. His fundamental work [19] is also the basis for our considerations. For further literature on this subject see [31], [23] and [30].

TheOREM 2.1. Let $\mathcal{C}$ be a semi-abelian category with enough injective objects, i.e., for every object there is a monohomomorphism into an injective object, and $R: \mathcal{C} \rightarrow \mathcal{L S}$ an additive functor. Then there is an associated sequence $R^{l}: \mathcal{C} \rightarrow \mathcal{L S}, l \in \mathbb{N}_{0}$, of additive functors (derived functors) such that every exact $\mathcal{C}$-sequence $0 \rightarrow F \rightarrow G \rightarrow H \rightarrow 0$ induces a natural exact $\mathcal{L S}$-sequence, the so-called long exact (cohomology) sequence:

$$
\begin{aligned}
0 \rightarrow R^{0}(F) \rightarrow R^{0}(G) \rightarrow R^{0}(H) \stackrel{\delta}{\rightarrow} R^{1}(F) \rightarrow R^{1}(G) & \rightarrow R^{1}(H) \\
& \stackrel{\delta}{\rightarrow} R^{2}(F) \rightarrow \cdots .
\end{aligned}
$$

The proof is as in [19, Proposition 2.1]. Note that V. P. Palamodov assumes that the functor $R$ is semi-injective, i.e., $R$ takes monohomomorphisms to monohomomorphisms, but this is neither needed for the definition of the derivatives nor for the deduction of the long exact sequence. Nevertheless, as it is of general interest regarding exactness, we will discuss this property immediately after introducing the derivatives of tensor product functors.

Let us briefly recall the definition of derivatives. Let $F$ be a $\mathcal{C}$-object and $0 \rightarrow F \stackrel{\varepsilon}{\rightarrow} F^{0} \stackrel{i^{0}}{\rightarrow} F^{1} \stackrel{i^{1}}{\rightarrow} \cdots$ an injective resolution of $F$, i.e., an exact $\mathcal{C}$-sequence with all $F^{l}$ injective. Applying the functor $R$ and passing to cohomology gives $R^{l}(F)$, i.e., $R^{l}(F)=\operatorname{ker} R\left(i^{l}\right) / \operatorname{im} R\left(i^{l-1}\right)$, where $i^{-1}:=0$. This definition is, up to isomorphism, independent of the choice of resolution.

Theorem 2.1 clearly implies the next remark, which allows for an abstract reformulation of the central question of Section 1.

Remark 2.2. Suppose $R \simeq R^{0}$ and let $F$ be a $\mathcal{C}$-object. Then $R$ is exact on all short exact sequences starting with $F$ if and only if $R^{1}(F)=0$.

This easy fact was used by D. Vogt and others (see $[26,4]$ ) to study exactness properties of the functor $L(E, \cdot): \mathcal{F S} \rightarrow \mathcal{L S}$ for Fréchet spaces $E$ by characterizing the vanishing of $\operatorname{Ext}^{1}(E, F)$, or equivalently the existence of a splitting in any extension of $E$ by $F$, in the so-called four standard cases (nuclear and Köthe cases).

Next we introduce the derivatives of locally convex tensor product functors.

Definition 2.3. For a locally convex space $E$ and a tensor norm $\alpha$ we consider the functor

$$
E \widetilde{\otimes}_{\alpha}: \mathcal{F} \mathcal{S} \rightarrow \mathcal{L S}, \quad F \mapsto E \widetilde{\otimes}_{\alpha} F, \quad f \mapsto \operatorname{id}_{E} \widetilde{\otimes}_{\alpha} f,
$$

and define $\operatorname{Tor}_{\alpha}^{l}(E, \cdot):=\left(E \widetilde{\otimes}_{\alpha} \cdot\right)^{l}: \mathcal{F S} \rightarrow \mathcal{L S}, l \in \mathbb{N}_{0}$. 
REMARK 2.4. The functor $L(E, \cdot)$ is left exact and therefore semi-injective. For a locally convex space $E$ the functor $E \widetilde{\otimes}_{\varepsilon}$. is also semi-injective ([10, Corollary 16.2.2]). For a $\pi$-space $E$ the functor $E \widetilde{\otimes}_{\pi} \cdot$ is semi-injective, which fails in the general case as Remark 1.6 shows. Generalizing the a.p. for Banach spaces (see Remark 1.6), we get an interesting sufficient condition for semi-injectivity. We say that a locally convex space $E$ has the general approximation property (g.a.p.) if for any locally convex space $F$ the canonical map $E \widetilde{\otimes}_{\pi} F \rightarrow E \widetilde{\otimes}_{\varepsilon} F$ is injective. Now let $E$ be a locally convex space with the g.a.p. and $f: F \rightarrow G$ a monohomomorphism between locally convex spaces. As $\operatorname{id}_{E} \widetilde{\otimes}_{\varepsilon} f$ is injective, the commutative diagram of Remark 1.6 implies the semi-injectivity of the functor $E \widetilde{\otimes}_{\pi} \cdot$. The g.a.p. always implies the a.p.; this may be found in [3], together with other interesting results on this property. If for all Banach spaces $X$ and $z \in E \widetilde{\otimes}_{\pi} X$ there is a compact subset $K \subseteq E$ with $z \in \overline{\Gamma\left(K \otimes B_{X}\right)}$, where the closure is taken in $E \widetilde{\otimes}_{\pi} X$, the two notions coincide. By a well-known result of A. Grothendieck the latter is true for Fréchet spaces $E$. If $E=\operatorname{ind}_{N} E_{N}$ is an (LB)-space such that

$$
E \widetilde{\otimes}_{\pi} X=\operatorname{ind}_{N}\left(E_{N} \widetilde{\otimes}_{\pi} X\right)
$$

for all Banach spaces $X$, the above condition is satisfied, again by Grothendieck's result. Interchangeability of tensor products with inductive limits in the above sense is of central interest for us. We will study it at a later point.

For an additive functor $R: \mathcal{F S} \rightarrow \mathcal{L S}$ and a Fréchet space $F$ we will now develop a general method for computing the group $R^{1}(F)$. Our central tool will be the long exact sequence. A first step towards the computation is

EXAMPLE 2.5. Consider the projective limit functor proj: $\mathcal{L S}^{\mathbb{N}} \rightarrow \mathcal{L S}$ from Example 1.3(i). Then for a projective $\mathcal{L} \mathcal{S}$-spectrum $\mathcal{F}=\left(F_{n}, \varrho_{m}^{n}\right)$ we have

$$
\begin{aligned}
\operatorname{proj}^{0} \mathcal{F} \cong \operatorname{proj} \mathcal{F}=\operatorname{ker}(\sigma(\mathcal{F})), \quad \operatorname{proj}^{1} \mathcal{F}=\operatorname{coker}(\sigma(\mathcal{F})), \\
\operatorname{proj}^{l} \mathcal{F}=0 \quad \text { for } l \geq 2,
\end{aligned}
$$

where the linear map $\sigma(\mathcal{F})=\sigma: \prod_{n} F_{n} \rightarrow \prod_{n} F_{n}$ is given by $x \mapsto$ $\varrho_{n+1}^{n}\left(x_{n+1}\right)-x_{n}$.

Moreover, if $\mathcal{F}=\left(F_{n}, \varrho_{m}^{n}\right)$ and $\mathcal{G}=\left(G_{n}, \phi_{m}^{n}\right)$ are equivalent projective spectra, i.e., there are sequences of natural numbers $\left(k_{n}\right)_{n},\left(l_{n}\right)_{n}$ with $n \leq$ $l_{n} \leq k_{n} \leq l_{n+1}$ and linear maps $u_{n}, v_{n}$ such that $u_{n} \circ v_{n+1}=\phi_{l_{n+1}}^{l_{n}}$ and $v_{n+1} \circ u_{n+1}=\varrho_{k_{n+1}}^{k_{n}}$, then we have $\operatorname{proj}^{l} \mathcal{F} \cong \operatorname{proj}^{l} \mathcal{G}$ for all $l$. Proofs for the above can be found in [19] and [31].

Definition 2.6. A projective spectrum $\mathcal{F}=\left(F_{n}, \varrho_{m}^{n}\right)$ of Banach spaces is called a fundamental system of Banach spaces for the Fréchet space proj $\mathcal{F}$ 
if $\mathcal{F}$ is equivalent to a reduced projective spectrum of Banach spaces, or equivalently, if for all $n$ there is an $m \geq n$ such that for all $k \geq m$ one has an inclusion $\varrho_{m}^{n}\left(F_{m}\right) \subseteq \overline{\varrho_{k}^{n}\left(F_{k}\right)}$, where the closure is taken in $F_{n}$.

For instance every nuclear Fréchet space has a fundamental system of $l_{p^{-}}$ spaces $(1 \leq p \leq \infty)$, which can easily be seen using nuclear representations of the connecting maps.

The following definition turns out to be very useful for computing right derivatives:

Definition 2.7. We call an additive functor $R: \mathcal{F S} \rightarrow \mathcal{L S} \sigma$-stable if the functor commutes with countable products in the sense that the morphism $p_{\mathcal{F}}: R\left(\prod_{n} F_{n}\right) \rightarrow \prod_{n} R\left(F_{n}\right)$ induced by the sequence of projections $\prod_{n} F_{n} \rightarrow F_{m}$ is a natural isomorphism, and $\sigma(R(\mathcal{F})) \circ p_{\mathcal{F}}=p_{\mathcal{F}} \circ R(\sigma(\mathcal{F}))$ for all projective $\mathcal{F} \mathcal{S}$-spectra $\mathcal{F}=\left(F_{n}, \varrho_{m}^{n}\right)$, where $R(\mathcal{F}):=\left(R\left(F_{n}\right), R\left(\varrho_{m}^{n}\right)\right)$.

EXAMPLE 2.8. For a locally convex space $E$ and a tensor norm $\alpha$ the functors $L(E, \cdot), E \widetilde{\otimes}_{\alpha} \cdot: \mathcal{F S} \rightarrow \mathcal{L S}$ are $\sigma$-stable.

Proof. We will give a proof for the tensor product functors. Due to [6, Korollar 2.12] the functor $E \widetilde{\otimes}_{\alpha}$. commutes with (countable) products. As the space $E \otimes_{\alpha} \prod F_{n}$ is dense in $E \widetilde{\otimes}_{\alpha} \prod F_{n}$, an easy calculation with elementary tensors gives the claim.

With the above definition we can formulate and prove our main theorem for computing derivatives.

TheOREm 2.9. Let $R: \mathcal{F S} \rightarrow \mathcal{L S}$ be a $\sigma$-stable functor. For all $l \in \mathbb{N}$ and fundamental systems of Banach spaces $\mathcal{F}=\left(F_{n}, \varrho_{m}^{n}\right)$ for a Fréchet space $F$ we have an exact $\mathcal{L S}$-sequence

$$
0 \rightarrow \operatorname{proj}^{1} R^{l-1}\left(F_{n}\right) \rightarrow R^{l}(F) \rightarrow \operatorname{proj} R^{l}\left(F_{n}\right) \rightarrow 0 .
$$

Proof. First we show that the derivatives $R^{l}$ are also $\sigma$-stable. For this, choose injective resolutions

$$
0 \rightarrow F_{n} \stackrel{\varepsilon_{n}}{\longrightarrow} F_{n}^{0} \stackrel{i_{n}^{0}}{\longrightarrow} F_{n}^{1} \stackrel{i_{n}^{1}}{\longrightarrow} \cdots
$$

which induce an injective resolution

$$
0 \rightarrow \prod F_{n} \stackrel{\left(\varepsilon_{n}\right)_{n}}{\longrightarrow} \prod F_{n}^{0} \stackrel{\left(i_{n}^{0}\right)_{n}}{\longrightarrow} \prod F_{n}^{1} \stackrel{\left(i_{n}^{1}\right)_{n}}{\longrightarrow} \cdots
$$

Further we find for all $n \in \mathbb{N}$ a unique (up to homotopy) morphism $\left(\left(\varrho_{n+1}^{n}\right)^{l}\right)_{l}$ which lifts $\varrho_{n+1}^{n}$, so that we can also consider the projective $\mathcal{F} \mathcal{S}$-spectra $\mathcal{F}^{l}:=\left(F_{n}^{l},\left(\varrho_{n+1}^{n}\right)^{l}\right), l \in \mathbb{N}_{0}$. The central observation is that the unique (up to homotopy) morphism $\left(\sigma^{l}\right)_{l}$, lifting $\sigma=\sigma(\mathcal{F})$, 


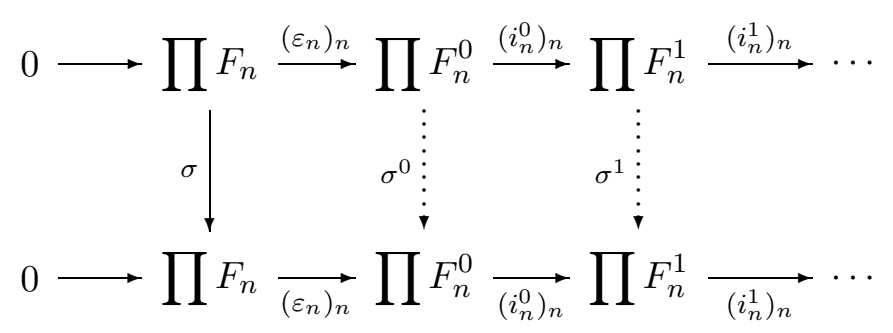

is given by $\sigma\left(\mathcal{F}^{l}\right)$ : with $\sigma\left(\mathcal{F}^{-1}\right):=\sigma(\mathcal{F}), i_{n}^{-1}:=\varepsilon_{n}, F_{n}^{-1}:=F_{n}$, we have

$$
\begin{aligned}
\sigma\left(\mathcal{F}^{l}\right) \circ\left(i_{n}^{l-1}\right)_{n}(x) & =\left(\left(\varrho_{n+1}^{n}\right)^{l} \circ i_{n+1}^{l-1}\left(x_{n+1}\right)-i_{n}^{l-1}\left(x_{n}\right)\right)_{n} \\
& =\left(i_{n}^{l-1} \circ\left(\varrho_{n+1}^{n}\right)^{l-1}\left(x_{n+1}\right)-i_{n}^{l-1}\left(x_{n}\right)\right)_{n} \\
& =\left(i_{n}^{l-1}\left(\left(\varrho_{n+1}^{n}\right)^{l-1}\left(x_{n+1}\right)-x_{n}\right)\right)_{n}=\left(i_{n}^{l-1}\right)_{n} \circ \sigma\left(\mathcal{F}^{l-1}\right)(x)
\end{aligned}
$$

for all $l \in \mathbb{N}_{0}$ and $x \in \prod F_{n}^{l-1}$. As $R$ is $\sigma$-stable, we may consider the following commutative diagram:

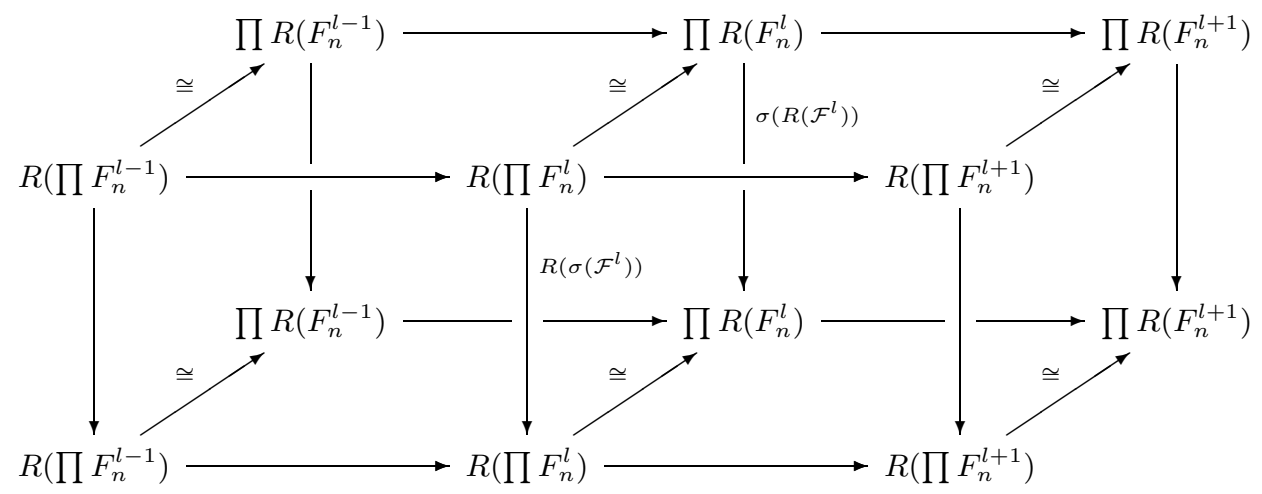

for all $l \in \mathbb{N}_{0}$, where $\sigma\left(\mathcal{F}^{-1}\right):=0, i_{n}^{-1}:=0$ and $F_{n}^{-1}:=0$. Passing to cohomology gives the $\sigma$-stability of the $R^{l}$.

Next, consider the exact sequence (called the canonical sequence)

$$
0 \rightarrow F \stackrel{\varrho}{\rightarrow} \prod F_{n} \stackrel{\sigma}{\rightarrow} \prod F_{n} \rightarrow 0
$$

where $\varrho(x):=\left(\varrho_{n}(x)\right)_{n}$ (see [26]). Applying the long exact sequence to this gives an exact $\mathcal{L} \mathcal{S}$-sequence

$$
\begin{aligned}
\cdots \rightarrow R^{l-1}\left(\prod F_{n}\right) \stackrel{R^{l-1}(\sigma)}{\longrightarrow} R^{l-1}\left(\prod F_{n}\right) \stackrel{\Delta}{\longrightarrow} R^{l}(F) \stackrel{R^{l}(\varrho)}{\longrightarrow} R^{l}\left(\prod F_{n}\right) \\
\stackrel{R^{l}(\sigma)}{\longrightarrow} R^{l}\left(\prod F_{n}\right) \rightarrow \cdots .
\end{aligned}
$$

From this, the $\sigma$-stability of the derivatives, and Example 2.5, we get the isomorphisms $\operatorname{ker} R^{l}(\varrho)=\operatorname{im} \Delta \cong \operatorname{coim} \Delta \cong \operatorname{coker} R^{l-1}(\sigma) \cong \operatorname{proj}^{1} R^{l-1}\left(F_{n}\right)$ and $\operatorname{im} R^{l}(\varrho)=\operatorname{ker} R^{l}(\sigma) \cong \operatorname{proj} R^{l}\left(F_{n}\right)$. The exactness of the sequences $0 \rightarrow \operatorname{ker} R^{l}(\varrho) \rightarrow R^{l}(F) \rightarrow \operatorname{im} R^{l}(\varrho) \rightarrow 0$ implies the theorem. 
REMARK 2.10. Another classical method for computing cohomology groups is by means of spectral sequences. Especially the Grothendieck spectral sequence (see [30, Theorem 5.8.3] and [19, Proposition 2.2] for the semi-abelian version) can be used to compute the derivatives of composed functors. Applying this spectral sequence to the composition $\mathcal{F} \mathcal{S}^{\mathbb{N}} \stackrel{R}{\rightarrow}$ $\mathcal{L} \mathcal{S}^{\mathbb{N}} \stackrel{\text { proj }}{\longrightarrow} \mathcal{L} \mathcal{S}$ and using the fact that $R$ commutes with countable products (so $R$ takes free projective spectra to free spectra), as well as $\operatorname{proj}^{2} \mathcal{X}=0$, gives for all $l \in \mathbb{N}$ an exact $\mathcal{L S}$-sequence

$$
0 \rightarrow \operatorname{proj}^{1} R^{l-1}\left(F_{n}\right) \rightarrow(\operatorname{proj} \circ R)^{l}(\mathcal{F}) \rightarrow \operatorname{proj} R^{l}\left(F_{n}\right) \rightarrow 0 .
$$

This however leaves the group $(\operatorname{proj} \circ R)^{l}(\mathcal{F})$ to be computed.

Theorem 2.9 becomes usable in cases of acyclicity:

Definition 2.11. We call a Fréchet space $F$

(a) $R$-acyclic if $R^{l}(F)=0$ for all $l \geq 1$,

(b) locally $R$-acyclic if $F$ has a fundamental system of $R$-acyclic Banach spaces,

(c) locally injective (resp. locally projective) if $F$ has a fundamental system of injective (resp. projective) Banach spaces.

For instance, injective Fréchet spaces are $R$-acyclic. Consequently, locally injective Fréchet spaces are locally $R$-acyclic. Nuclear Fréchet spaces and quasinormable Köthe spaces are locally injective.

REMARK 2.12. For $R$-acyclic Fréchet spaces $F$ the identity $R^{0}(F)=$ $R(F)$ holds.

Proof. By a result of A. Grothendieck, for the definition of the derivatives one may use $R$-acyclic resolutions instead of injective ones (cf. [30, Exercise 2.4.3]). Applying this to $0 \rightarrow F \rightarrow F \rightarrow 0 \rightarrow 0 \rightarrow \cdots$ gives the claim.

Corollary 2.13. Let $R: \mathcal{F S} \rightarrow \mathcal{L S}$ be a $\sigma$-stable functor and $F$ a Fréchet space with a fundamental system of Banach spaces $\mathcal{F}=\left(F_{n}, \varrho_{m}^{n}\right)$. Then

(a) $R^{1}(F)=0 \Rightarrow \operatorname{proj}^{1} R^{0}\left(F_{n}\right)=0$.

(b) $R^{l}(F)=0$ for $l \geq 2$ and $R^{1}(F)=\operatorname{proj}^{1} R\left(F_{n}\right)$ if $F$ is locally $R$ acyclic.

Proof. (a) and (b) follow from Theorem 2.9 and the above remark. Note that $\operatorname{proj}^{l}$ depends only on the equivalence class of the projective spectrum considered (Example 2.5).

\section{First examples for derivatives of tensor product functors.} Before deducing necessary and sufficient conditions for the vanishing of $\operatorname{Tor}_{\alpha}^{1}(E, F)$ in the next section and applying them to standard cases, we will 
give some simple examples which may contribute to a better understanding of the subject.

REMARK 3.1. Let $E$ be a locally convex space with the a.p. Then the functor $E \widetilde{\otimes}_{\varepsilon} \cdot: \mathcal{F S} \rightarrow \mathcal{L S}$ is left exact. In particular, $\operatorname{Tor}_{\varepsilon}^{0}(E, F)=E \widetilde{\otimes}_{\varepsilon} F$ for any Fréchet space $F$.

Proof. First we may assume that $E$ is complete as $\widetilde{E}$ also has the a.p. and $E \widetilde{\otimes}_{\varepsilon} \cdot \simeq \widetilde{E} \widetilde{\otimes}_{\varepsilon} \cdot$. The assertion now follows from the coincidence of the $\varepsilon$-tensor product functor with the $\varepsilon$-product functor (see $[10,18.1 .8]$ ).

For the projective tensor product the situation is quite different:

EXAmPLE 3.2 . Let $\mathbb{K}=\mathbb{R}$. Then $l_{2} \widetilde{\otimes}_{\pi} l_{2}$ is a proper linear subspace of $\operatorname{Tor}_{\pi}^{0}\left(l_{2}, l_{2}\right)$ and the functor $l_{2} \widetilde{\otimes}_{\pi} \cdot: \mathcal{F S} \rightarrow \mathcal{L S}$ is not left exact.

Proof. Let $0 \rightarrow l_{2} \stackrel{\varepsilon}{\rightarrow} F^{0} \stackrel{i^{0}}{\rightarrow} F^{1} \rightarrow \cdots$ be an injective resolution of $l_{2}$, where the $F^{l}$ are injective Banach spaces. Consider the commutative diagram

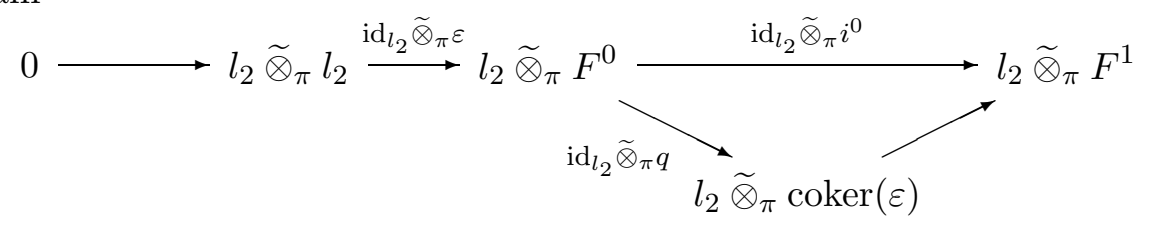

where $q: F^{0} \rightarrow \operatorname{coker}(\varepsilon)$ is the canonical map. Note that the functor $l_{2} \widetilde{\otimes}_{\pi}$. is semi-injective (Remark 2.4). By Example 1.3(ii) and Corollary 1.4(a) we have

$$
\begin{aligned}
\operatorname{Tor}_{\pi}^{0}\left(l_{2}, l_{2}\right) & =\operatorname{ker}\left(\operatorname{id}_{l_{2}} \widetilde{\otimes}_{\pi} i^{0}\right)=\operatorname{ker}\left(\operatorname{id}_{l_{2}} \widetilde{\otimes}_{\pi} q\right)=\left(\operatorname{ker}\left(\operatorname{id}_{l_{2}} \otimes_{\pi} q\right)\right)^{\sim} \\
& =\left(\operatorname{im}\left(\operatorname{id}_{l_{2}} \otimes_{\pi} \varepsilon\right) \tilde{)}=\overline{\operatorname{im}\left(\operatorname{id}_{l_{2}} \otimes_{\pi} \varepsilon\right)} l_{2} \widetilde{\otimes}_{\pi} F^{0} .\right.
\end{aligned}
$$

So it suffices to show that $\mathrm{id}_{l_{2}} \widetilde{\otimes}_{\pi} \varepsilon$ does not have closed range or equivalently is not a monohomomorphism. Assume the opposite. Let $\varphi$ be the linear map corresponding to the bilinear form $(x, y) \mapsto\langle x, y\rangle$ on the space $l_{2} \widetilde{\otimes}_{\pi} l_{2}$. By assumption and the Hahn-Banach theorem we find a continuous extension $\phi$ of $\varphi$ to the space $l_{2} \widetilde{\otimes}_{\pi} F^{0}$. Setting $P(y)=\left(x^{\prime} \mapsto \phi\left(x^{\prime} \otimes y\right)\right)$ gives, by the Riesz representation theorem, a continuous projection $P$ from $F^{0}$ onto $l_{2}=l_{2}^{\prime}$. Consequently, $l_{2}$ is a complemented subspace of an injective Banach space and therefore it is also injective, a contradiction.

Later we shall need the following result, which follows from Remark 2.2 and Corollary 1.8:

Proposition 3.3. Let $E$ be an $\alpha$-space, where $\alpha=\pi$ or $\varepsilon$. Then for all exact $\mathcal{F S}$-sequences $0 \rightarrow F \rightarrow G \rightarrow H \rightarrow 0$ the tensored sequence $0 \rightarrow E \widetilde{\otimes}_{\alpha} F \rightarrow E \widetilde{\otimes}_{\alpha} G \rightarrow E \widetilde{\otimes}_{\alpha} H \rightarrow 0$ is topologically exact if and only if $\operatorname{Tor}_{\alpha}^{1}(E, F)=0$. 
A similar argument gives

Proposition 3.4. A Fréchet space $E$ is an $\alpha$-space $(\alpha=\pi$ or $\varepsilon)$ if and only if $\operatorname{Tor}_{\alpha}^{0}(E, \cdot) \simeq E \widetilde{\otimes}_{\alpha} \cdot$ and $\operatorname{Tor}_{\alpha}^{1}(E, \cdot)=0$.

As a direct consequence of Proposition 3.4 and the fact that $l_{1}$ has the a.p. and is not an $\varepsilon$-space we get:

Example 3.5. There is a Fréchet space $F$ (even a Banach space) with $\operatorname{Tor}_{\pi}^{1}\left(l_{1}, F\right)=0 \neq \operatorname{Tor}_{\varepsilon}^{1}\left(l_{1}, F\right)$.

Since $l_{1}$ is a $\pi$-Banach space satisfying the a.p. and $l_{\infty}$ is not nuclear, we have:

EXAMPLE 3.6. $\operatorname{Tor}_{\pi}^{0}\left(l_{1}, l_{\infty}\right)=l_{1} \widetilde{\otimes}_{\pi} l_{\infty} \neq l_{1} \widetilde{\otimes}_{\varepsilon} l_{\infty}=\operatorname{Tor}_{\varepsilon}^{0}\left(l_{1}, l_{\infty}\right)$. On the other hand, $l_{\infty}$ is injective, so $\operatorname{Tor}_{\pi}^{1}\left(l_{1}, l_{\infty}\right)=0=\operatorname{Tor}_{\varepsilon}^{1}\left(l_{1}, l_{\infty}\right)$.

EXAmple 3.7. There are Fréchet spaces $F$ with $\operatorname{Tor}_{\pi}^{1}\left(l_{1}, F\right)=0 \neq$ $\operatorname{Ext}^{1}\left(l_{\infty}, F\right)$. Otherwise the functor $L\left(l_{\infty}, \cdot\right): \mathcal{F S} \rightarrow \mathcal{L S}$ would be exact and $l_{\infty}$ projective, which is not the case.

The following example is a reformulation of results of W. Kaballo and D. Vogt:

EXAMPLE 3.8. Let $E=\operatorname{ind}_{N} E_{N}$ be a compact regular $(L B)$-space, i.e., every compact subset of $E$ is localized in one step and compact there. Further assume that each $E_{N}$ has the a.p. Then $\operatorname{Tor}_{\varepsilon}^{1}\left(E, \mathcal{L}_{\infty}\right)=0$.

Proof. First note that $E$ also has the a.p. ([10, Proposition 18.2.5]), so the functor $E \widetilde{\otimes}_{\varepsilon} \cdot: \mathcal{F S} \rightarrow \mathcal{L S}$ is left exact. The assertion now follows from $[12,1.10]$ and the long exact sequence.

As a consequence of $[11,2.13]$, one obtains an example dual to Example 3.8:

EXAMPLE 3.9. Let $E$ be a compact regular (LB)-space with $\mathcal{L}_{\infty}$-steps. Then $\operatorname{Tor}_{\varepsilon}^{1}(E, X)=0$ for all Banach spaces $X$.

4. Necessary and sufficient conditions for $\operatorname{Tor}_{\alpha}^{1}(E, F)=0$. Now let us deduce necessary conditions for the vanishing of $\operatorname{Tor}_{\alpha}^{1}(E, F)$. In relevant cases, Corollary 2.13 says that for a tensor norm $\alpha$, a locally convex space $E$ and a Fréchet space $F$ with a fundamental system of Banach spaces $\left(F_{n}, \varrho_{m}^{n}\right), \operatorname{Tor}_{\alpha}^{1}(E, F)=0$ implies $\operatorname{proj}^{1} E \widetilde{\otimes}_{\alpha} F_{n}=0$. Applying the theorem of Palamodov-Retakh (see [19, Theorem 5.4]) we get first necessary conditions for $\operatorname{Tor}_{\alpha}^{1}(E, F)=0$, provided that the assumptions of that theorem are satisfied. In other words, we are interested in (bounded) Banach discs in $E \widetilde{\otimes}_{\alpha} F_{n}$. For the rest of the section we will assume (unless otherwise stated) the following setup: 
Setting 4.1. Let $\alpha$ be a tensor norm, $E=\operatorname{ind}_{N} E_{N}$ a complete (LB)space with connecting maps $i_{N}^{N+1}: E_{N} \hookrightarrow E_{N+1}, i_{N}: E_{N} \hookrightarrow E$ and without loss of generality $B_{1}:=B_{E_{1}} \subseteq B_{2}:=B_{E_{2}} \subseteq \cdots$ a fundamental sequence of closed and bounded subsets of $E$. We assume that $E$ is a proper $(L B)$-space, i.e. $E$ is not a Banach space. For a Fréchet space $F$ with a fundamental system of Banach spaces $\left(F_{n}, \varrho_{m}^{n}\right)$ let $U_{n}:=B_{F_{n}}, n \in \mathbb{N}$. We further assume

(a) $\operatorname{Tor}_{\alpha}^{1}(E, F)=0 \Rightarrow \operatorname{proj}^{1} E \widetilde{\otimes}_{\alpha} F_{n}=0$

and for a possibly different fundamental system of Banach spaces one of the conditions

$(\mathrm{b} 1) \operatorname{ind}_{N}\left(E_{N} \otimes_{\alpha} F_{n}\right)=E \otimes_{\alpha} F_{n}$ and each $F_{n}$ has the bounded approximation property (b.a.p.), i.e., there is an equicontinuous net of finite-dimensional operators on $F_{n}$ which converge pointwise to $\operatorname{id}_{F_{n}}$,

(b2) $\operatorname{ind}_{N}\left(E_{N} \widetilde{\otimes}_{\alpha} F_{n}\right)=E \widetilde{\otimes}_{\alpha} F_{n}$ and either all $E_{N}$ or all $F_{n}$ have the a.p.

Our ultimate goal is to deduce an evaluable necessary condition for the vanishing of $\operatorname{proj}^{1} E \widetilde{\otimes}_{\alpha} F_{n}$ of the following type:

For all $n$ there is an $N$ and an $m \geq n$ such that for all $M \geq N$, $k \geq m$ and $\varepsilon>0$ we can find a $K \geq M$ and an $S>0$ such that for all $x^{\prime} \in E^{\prime}\left(x^{\prime} \in E_{K}^{\prime}\right.$, respectively) we have

$$
\left\|x^{\prime}\right\|_{M}^{*} \varrho_{m}^{n}\left(U_{m}\right) \subseteq S\left\|x^{\prime}\right\|_{K}^{*} \varrho_{k}^{n}\left(U_{k}\right)+\varepsilon\left\|x^{\prime}\right\|_{N}^{*} U_{n},
$$

where $\left\|x^{\prime}\right\|_{L}^{*}:=\sup _{x \in B_{L}}\left|x^{\prime}(x)\right|$ (for $L \leq K$, respectively).

First, note that we assume (LB)-spaces to be injective. The latter is always satisfied in the case of the projective and injective tensor product, as the following remark shows (for arbitrary tensor norms see [2, 21.7]).

Remark 4.2. Let $\alpha=\pi$ or $\alpha=\varepsilon$ and let $X$ be a Banach space. For $\alpha=\pi$ we require that each $E_{N}$ or $X$ has the a.p. Then the maps $E_{N} \widetilde{\otimes}_{\alpha} X \rightarrow$ $E \widetilde{\otimes}_{\alpha} X$ and $E_{N} \widetilde{\otimes}_{\alpha} X \rightarrow E_{N+1} \widetilde{\otimes}_{\alpha} X$ are injective. Consequently, so also are $E_{N} \otimes_{\alpha} X \rightarrow E \otimes_{\alpha} X$ and $E_{N} \otimes_{\alpha} X \rightarrow E_{N+1} \otimes_{\alpha} X$.

Proof. Only for $\alpha=\pi$ is a proof needed. By assumption, the maps $E_{N} \widetilde{\otimes}_{\pi} X \rightarrow E_{N} \widetilde{\otimes}_{\varepsilon} X$ are injective. The assertion is now immediate due to commutative diagrams as in Remark 1.6.

Let us give some examples for Setting 4.1. Properties (b1) and (b2) are studied further in $[15,16,9,21]$.

EXAMPLE 4.3.

(1) For a nuclear Fréchet space $F$ it suffices to consider the projective tensor product (Proposition 5.5). As $F$ is locally injective, Corollary 2.13 gives $\operatorname{Tor}_{\pi}^{1}(E, F)=\operatorname{proj}^{1} E \widetilde{\otimes}_{\pi} F_{n}$ for all fundamental sys- 
tems of Banach spaces $\left(F_{n}, \varrho_{m}^{n}\right)$ for $F$. On the other hand, $F$ also has fundamental systems of $l_{1}$-spaces. Due to [18, Theorem 5] we have $\operatorname{ind}_{N}\left(E_{N} \widetilde{\otimes}_{\pi} l_{1}\right)=E \widetilde{\otimes}_{\pi} l_{1}$ for all regular (LB)-spaces $E$. So (a) and (b2) are satisfied.

(2) Let $\alpha=\varepsilon$ and let $F$ be a Fréchet space with a fundamental system consisting of $\mathcal{L}_{\infty}$-spaces. A short calculation using [12, Satz 1.9(i)] shows that $\operatorname{Tor}_{\varepsilon}^{0}\left(E, \mathcal{L}_{\infty}\right)=E \widetilde{\otimes}_{\varepsilon} \mathcal{L}_{\infty}$. From this we get (a) and due to $[20,11.4 .45]$ we have $\operatorname{ind}_{N}\left(E_{N} \otimes_{\varepsilon} \mathcal{L}_{\infty}\right)=E \otimes_{\varepsilon} \mathcal{L}_{\infty}$. So (b1) also holds. For compact regular (LB)-spaces $E$ we even have (b2) ([7, Corollary 4.4]).

(3) Let $\alpha$ be a tensor norm and $E$ an (LB)-space with a partition of unity. Then (b1) holds (see [6, Korollar 2.14] for the result and the terminology).

The interchangeability condition (b2) is quite a strong one, but very useful for computations, while (b1) is always true for the projective tensor product $([10,15.5 .4])$. Therefore we will work with Setting (a) and (b1) and return to (b2) later.

By [20, Proposition 11.5.7] the space $\operatorname{ind}_{N}\left(E_{N} \otimes_{\alpha} F_{n}\right)=E \otimes_{\alpha} F_{n}$ is large in $E \widetilde{\otimes}_{\alpha} F_{n}$ for all $n$, i.e., every bounded subset of $E \widetilde{\otimes}_{\alpha} F_{n}$ is contained in the closure of a bounded subset of $E \otimes_{\alpha} F_{n}$. Consequently, $\left(\overline{B_{E_{N} \otimes_{\alpha} F_{n}}} E \tilde{\otimes}_{\alpha} F_{n}\right)_{n}$ is a fundamental sequence of bounded subsets of $E \widetilde{\otimes}_{\alpha} F_{n}$.

Let $C_{n, 1}:=1$. As the maps $i_{N}^{N+1} \otimes_{\alpha} \operatorname{id}_{F_{n}}$ are continuous we inductively find a sequence of constants $C_{n, N} \geq N$ with $i_{N}^{N+1} \otimes_{\alpha} \operatorname{id}_{F_{n}}\left(C_{n, N} B_{E_{N} \otimes_{\alpha} F_{n}}\right) \subseteq$ $C_{n, N+1} B_{E_{N+1} \otimes_{\alpha} F_{n}}$. Setting

$$
B_{n, N}:=C_{n, N} \overline{B_{E_{N} \otimes_{\alpha} F_{n}}} E \tilde{\otimes}_{\alpha} F_{n}
$$

gives an increasing sequence of bounded Banach discs in $E \widetilde{\otimes}_{\alpha} F_{n}$ which cover the whole space, such that for all $N$ there exists an $\widetilde{N}$ with the property $\operatorname{id}_{E} \widetilde{\otimes}_{\alpha} \varrho_{n+1}^{n}\left(B_{n+1, N}\right) \subseteq B_{n, \widetilde{N}}$. The first assertions are clear from the definitions while the last follows from

$$
\begin{aligned}
& \operatorname{id}_{E} \widetilde{\otimes}_{\alpha} \varrho_{n+1}^{n}\left(B_{n+1, N}\right) \subseteq \overline{\operatorname{id}_{E} \widetilde{\otimes}_{\alpha} \varrho_{n+1}^{n}\left(C_{n+1, N} B_{E_{N} \otimes_{\alpha} F_{n+1}}\right)} E \widetilde{\otimes}_{\alpha} F_{n} \\
& =\overline{\operatorname{id}_{E_{N}} \otimes_{\alpha} \varrho_{n+1}^{n}\left(C_{n+1, N} B_{E_{N} \otimes_{\alpha} F_{n+1}}\right)} E \widetilde{\otimes}_{\alpha} F_{n}
\end{aligned}
$$

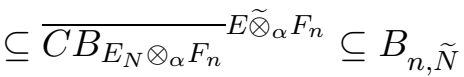

for suitable $C=C(N, n)>0, \widetilde{N}=\widetilde{N}(n, N)$. We have thus verified the assumptions of [19, Theorem 5.4] and so we obtain

LEMmA 4.4. With the above terminology, $\operatorname{proj}^{1} E \widetilde{\otimes}_{\alpha} F_{n}=0$ implies $\left(\widetilde{S}_{2}\right) \quad$ For all $n$ there is an $N$ and an $m \geq n$ such that for all $k \geq m$,

$$
\operatorname{id}_{E} \widetilde{\otimes}_{\alpha} \varrho_{m}^{n}\left(E \widetilde{\otimes}_{\alpha} F_{m}\right) \subseteq \operatorname{id}_{E} \widetilde{\otimes}_{\alpha} \varrho_{k}^{n}\left(E \widetilde{\otimes}_{\alpha} F_{k}\right)+B_{n, N} .
$$


Next we want to refine $\left(\widetilde{S}_{2}\right)$. We shall achieve this step by step with a series of technical results (see also $[26,27]$ ).

LEMMA 4.5. $\left(\widetilde{S}_{2}\right)$ implies

$\left(S_{2}\right) \quad$ For all $n$ there is an $N$ and an $m \geq n$ such that for all $M \geq N$ and $k \geq m$ we can find $a K \geq M$ and an $S>0$ with

$$
\operatorname{id}_{E} \widetilde{\otimes}_{\alpha} \varrho_{m}^{n}\left(B_{m, M}\right) \subseteq S\left(\operatorname{id}_{E} \widetilde{\otimes}_{\alpha} \varrho_{k}^{n}\left(B_{k, K}\right)+B_{n, N}\right) .
$$

Proof. This is a straightforward application of Grothendieck's factorization theorem (see [26, Lemma 2.2] or [27, Lemma 2.3]).

LEMMA 4.6. $\left(S_{2}\right)$ implies the condition

$\left(S_{2}^{\prime}\right) \quad$ For all $n$ there is an $N$ and an $m \geq n$ such that for all $M \geq N$ and $k \geq m$ we can find $a K \geq M$ and an $S>0$ such that for all $x^{\prime} \in E^{\prime}$,

$$
\left\|x^{\prime}\right\|_{M}^{*} \varrho_{m}^{n}\left(U_{m}\right) \subseteq S\left(\left\|x^{\prime}\right\|_{K}^{*} \varrho_{k}^{n}\left(U_{k}\right)+\left\|x^{\prime}\right\|_{N}^{*} U_{n}\right),
$$

where $\left\|x^{\prime}\right\|_{L}^{*}:=\sup _{x \in B_{L}}\left|x^{\prime}(x)\right|$.

Proof. We may assume $x^{\prime} \in E^{\prime} \backslash\{0\}$. By definition for all $M \in \mathbb{N}$ we can find an $x_{0}=x_{0}(M) \in B_{M}$ with $\left\|x^{\prime}\right\|_{M}^{*} \leq 2 x^{\prime}\left(x_{0}\right)$. For $y \in U_{m}$ we have $x_{0} \otimes_{\alpha} y \in B_{E_{M} \otimes_{\alpha} F_{m}}$ and therefore $x_{0} \widetilde{\otimes}_{\alpha} y \in C_{m, M} \overline{B_{E_{M} \otimes_{\alpha} F_{m}}} E \widetilde{\otimes}_{\alpha} F_{m}=B_{m, M}$. Using $\left(S_{2}\right)$ we get

$$
\begin{aligned}
\left\|x^{\prime}\right\|_{M}^{*} \varrho_{m}^{n}\left(U_{m}\right) & \subseteq 2 x^{\prime}\left(x_{0}\right) \varrho_{m}^{n}\left(U_{m}\right) \subseteq 2 x^{\prime} \widetilde{\otimes}_{\alpha} \operatorname{id}_{F_{n}}\left(x_{0} \widetilde{\otimes}_{\alpha} \varrho_{m}^{n}\left(U_{m}\right)\right) \\
& \subseteq 2 x^{\prime} \widetilde{\otimes}_{\alpha} \operatorname{id}_{F_{n}}\left(\operatorname{id}_{E} \widetilde{\otimes}_{\alpha} \varrho_{m}^{n}\left(B_{m, M}\right)\right) \\
& \subseteq 2 S x^{\prime} \widetilde{\otimes}_{\alpha} \operatorname{id}_{F_{n}}\left(\operatorname{id}_{E} \widetilde{\otimes}_{\alpha} \varrho_{k}^{n}\left(B_{k, K}\right)+B_{n, N}\right) \\
& =2 S x^{\prime} \widetilde{\otimes}_{\alpha} \varrho_{k}^{n}\left(B_{k, K}\right)+2 S x^{\prime} \widetilde{\otimes}_{\alpha} \operatorname{id}_{F_{n}}\left(B_{n, N}\right) .
\end{aligned}
$$

Now we prove the inclusions

(i) $x^{\prime} \widetilde{\otimes}_{\alpha} \operatorname{id}_{F_{n}}\left(B_{E_{N} \otimes_{\alpha} F_{n}}\right) \subseteq\left\|x^{\prime}\right\|_{N}^{*} U_{n}$,

(ii) $x^{\prime} \widetilde{\otimes}_{\alpha} \varrho_{k}^{n}\left(\overline{B_{E_{K} \otimes_{\alpha} F_{k}}} E \widetilde{\otimes}_{\alpha} F_{k}\right) \subseteq\left\|x^{\prime}\right\|_{K}^{*} \varrho_{k}^{n}\left(U_{k}\right)$.

Indeed, $x^{\prime} \widetilde{\otimes}_{\alpha} \operatorname{id}_{F_{n}}\left(B_{E_{N} \otimes_{\alpha} F_{n}}\right)=\left.x^{\prime}\right|_{E_{N}} \otimes_{\alpha} \operatorname{id}_{F_{n}}\left(B_{E_{N} \otimes_{\alpha} F_{n}}\right)$ is contained in $\left\|\left.x^{\prime}\right|_{E_{N}} \otimes_{\alpha} \operatorname{id}_{F_{n}}\right\| U_{n} \subseteq\left\|x^{\prime}\right\|_{N}^{*} \cdot 1 \cdot U_{n}$, which proves (i). Next, we consider the maps $\varrho_{k}^{n}: F_{k} \rightarrow F_{n}$ and endow $\varrho_{k}^{n}\left(F_{k}\right)$ with the quotient norm, so that $\varrho_{k}^{n}\left(U_{k}\right)$ is the unit ball in $\varrho_{k}^{n}\left(F_{k}\right)$ and $\left\|\varrho_{k}^{n}: F_{k} \rightarrow \varrho_{k}^{n}\left(F_{k}\right)\right\|=1$. We obtain

$$
\begin{aligned}
x^{\prime} \widetilde{\otimes}_{\alpha} \varrho_{k}^{n}\left(\bar{B}_{E_{K} \otimes_{\alpha} F_{k}} \tilde{\otimes}_{\alpha} F_{k}\right) & =\left(\operatorname{id}_{\mathbb{K}} \widetilde{\otimes}_{\alpha} \varrho_{k}^{n}\right) \circ\left(x^{\prime} \widetilde{\otimes}_{\alpha} \operatorname{id}_{F_{k}}\right)\left({\overline{B_{E_{K} \otimes_{\alpha} F_{k}}}}^{E \widetilde{\otimes}_{\alpha} F_{k}}\right) \\
& \subseteq \varrho_{k}^{n}\left(\left\|x^{\prime}\right\|_{K}^{*} \cdot 1 \cdot U_{k}\right)=\left\|x^{\prime}\right\|_{K}^{*} \varrho_{k}^{n}\left(U_{k}\right),
\end{aligned}
$$

which is (ii). Together, (i) and (ii) give

$$
\begin{aligned}
\left\|x^{\prime}\right\|_{M}^{*} \varrho_{m}^{n}\left(U_{m}\right) & \subseteq 2 S x^{\prime} \widetilde{\otimes}_{\alpha} \varrho_{k}^{n}\left(B_{k, K}\right)+2 S x^{\prime} \widetilde{\otimes}_{\alpha} \operatorname{id}_{F_{n}}\left(B_{n, N}\right) \\
& =2 S\left(x^{\prime} \widetilde{\otimes}_{\alpha} \varrho_{k}^{n}\left(C_{k, K} \overline{B_{E_{K} \otimes_{\alpha} F_{k}}}\right)+x^{\prime} \widetilde{\otimes}_{\alpha} \operatorname{id}_{F_{n}}\left(C_{n, N} \overline{B_{E_{N} \otimes_{\alpha} F_{n}}}\right)\right)
\end{aligned}
$$




$$
\begin{aligned}
& \subseteq 2 S\left(C_{k, K}\left\|x^{\prime}\right\|_{K}^{*} \varrho_{k}^{n}\left(U_{k}\right)+C_{n, N}\left\|x^{\prime}\right\|_{N}^{*} U_{n}\right) \\
& \subseteq \widetilde{S}\left(\left\|x^{\prime}\right\|_{K}^{*} \varrho_{k}^{n}\left(U_{k}\right)+\left\|x^{\prime}\right\|_{N}^{*} U_{n}\right),
\end{aligned}
$$

where $\widetilde{S}=\max \left\{2 S C_{k, K}, 2 S C_{n, N}\right\}$, proving the claim.

The following condition $\left(S_{2}^{\prime}\right)_{0}$ is central to our further considerations.

Proposition 4.7. The condition

$\left(S_{2}^{\prime}\right)_{0} \quad$ For all $n$ there is an $N$ and an $m \geq n$ such that for all $M \geq N$, $k \geq m$ and $\varepsilon>0$ there is $K \geq M$ and an $S>0$ such that for all $x^{\prime} \in E^{\prime}$ we have

$$
\left\|x^{\prime}\right\|_{M}^{*} \varrho_{m}^{n}\left(U_{m}\right) \subseteq S\left\|x^{\prime}\right\|_{K}^{*} \varrho_{k}^{n}\left(U_{k}\right)+\varepsilon\left\|x^{\prime}\right\|_{N}^{*} U_{n}
$$

is necessary for $\left(S_{2}^{\prime}\right)$.

Proof. First we deduce the following condition from $\left(S_{2}^{\prime}\right)$ :

(Q) For all $n$ there is an $\widetilde{n} \geq n$ such that for all $k \geq \widetilde{n}$ and $\varepsilon>0$ we can find an $\widetilde{S}>0$ with

$$
\varrho_{\widetilde{n}}^{n}\left(U_{\widetilde{n}}\right) \subseteq \widetilde{S} \varrho_{k}^{n}\left(U_{k}\right)+\varepsilon U_{n} .
$$

Choose $N$ and $m \geq n$ using $\left(S_{2}^{\prime}\right)$ and set $\widetilde{n}:=m$. As $E$ is a proper regular (LB)-space, $E_{b}^{\prime}$ is a proper Fréchet space. So there is an $M \geq N$ with $\|\cdot\|_{M}^{*} \nsim\|\cdot\|_{N}^{*}$. Consequently,

$$
\inf _{\left\|x^{\prime}\right\|_{M}^{*} \neq 0} \frac{\left\|x^{\prime}\right\|_{N}^{*}}{\left\|x^{\prime}\right\|_{M}^{*}}=0
$$

and we can find a sequence $\left(x_{L}^{\prime}\right)_{L}$ in $E^{\prime}$ with $\left\|x_{L}^{\prime}\right\|_{M}^{*} \neq 0$ for all $L$ and $\lim _{L \rightarrow \infty}\left\|x_{L}^{\prime}\right\|_{N}^{*} /\left\|x_{L}^{\prime}\right\|_{M}^{*}=0$. Let $k \geq \widetilde{n}$ and $\varepsilon>0$ be arbitrary. Then $\left(S_{2}^{\prime}\right)$ implies the existence of a $K \geq M$ and an $S>0$ such that for all $L$ we have

$$
\left\|x_{L}^{\prime}\right\|_{M}^{*} \varrho_{\widetilde{n}}^{n}\left(U_{\widetilde{n}}\right) \subseteq S\left(\left\|x_{L}^{\prime}\right\|_{K}^{*} \varrho_{k}^{n}\left(U_{k}\right)+\left\|x_{L}^{\prime}\right\|_{N}^{*} U_{n}\right) .
$$

Now we choose $L$ in such a way that $\left\|x_{L}^{\prime}\right\|_{N}^{*} /\left\|x_{L}^{\prime}\right\|_{M}^{*}<\varepsilon / S$. With $\widetilde{S}:=$ $S\left\|x_{L}^{\prime}\right\|_{K}^{*} /\left\|x_{L}^{\prime}\right\|_{M}^{*}$ we get the condition $(Q)$.

Next let us prove $\left(S_{2}^{\prime}\right)_{0}$. Let $n$ be arbitrary. We choose $\widetilde{n} \geq n$ according to $(Q)$ and apply $\left(S_{2}^{\prime}\right)$ to $\widetilde{n}$, which gives an $N$ and an $m \geq \widetilde{n}$. For given $M \geq N, k \geq m$ and $\varepsilon>0$ the condition $\left(S_{2}^{\prime}\right)$ implies the existence of a $K \geq M$ and an $\widehat{S}>0$ such that for all $x^{\prime} \in E^{\prime}$ we have

$$
\left\|x^{\prime}\right\|_{M}^{*} \varrho_{m}^{\widetilde{n}}\left(U_{m}\right) \subseteq \widehat{S}\left(\left\|x^{\prime}\right\|_{K}^{*} \varrho_{k}^{\widetilde{n}}\left(U_{k}\right)+\left\|x^{\prime}\right\|_{N}^{*} U_{\widetilde{n}}\right) .
$$

Applying $(Q)$ to $k$ and $\varepsilon / \widehat{S}$ gives an $\widetilde{S}$ with $\varrho_{\widetilde{n}}^{n}\left(U_{\widetilde{n}}\right) \subseteq \widetilde{S} \varrho_{k}^{n}\left(U_{k}\right)+(\varepsilon / \widehat{S}) U_{n}$. From this and (3) we get 


$$
\begin{aligned}
\left\|x^{\prime}\right\|_{M}^{*} \varrho_{m}^{n}\left(U_{m}\right) & \subseteq \widehat{S}\left(\left\|x^{\prime}\right\|_{K}^{*} \varrho_{k}^{n}\left(U_{k}\right)+\left\|x^{\prime}\right\|_{N}^{*} \varrho_{\tilde{n}}^{n}\left(U_{\widetilde{n}}\right)\right) \\
& \subseteq \widehat{S}\left(\left\|x^{\prime}\right\|_{K}^{*} \varrho_{k}^{n}\left(U_{k}\right)+\left\|x^{\prime}\right\|_{N}^{*}\left(\widetilde{S} \varrho_{k}^{n}\left(U_{k}\right)+(\varepsilon / \widehat{S}) U_{n}\right)\right) \\
& \subseteq \widehat{S}(1+\widetilde{S})\left\|x^{\prime}\right\|_{K}^{*} \varrho_{k}^{n}\left(U_{k}\right)+\varepsilon\left\|x^{\prime}\right\|_{N}^{*} U_{n}
\end{aligned}
$$

Setting $S:=\widehat{S}(1+\widetilde{S})$ implies $\left(S_{2}^{\prime}\right)_{0}$.

The following result is of technical significance as it will allow us to weaken our assumptions on the space $E$ when proving that certain necessary conditions are also sufficient.

THEOREM 4.8. If F has a fundamental system of Banach spaces $\left(F_{n}, \varrho_{m}^{n}\right)$ such that the interchangeability property of Setting 4.1(b2) is satisfied, we even get instead of $\left(S_{2}^{\prime}\right)_{0}$ :

$\left(S_{2}^{K \prime}\right)_{0} \quad$ For all $n$ there is an $N$ and an $m \geq n$ such that for all $M \geq N$, $k \geq m$ and $\varepsilon>0$ we can find $a K \geq M$ and an $S>0$ such that for all $x^{\prime} \in E_{K}^{\prime}$ we have

$$
\left\|x^{\prime}\right\|_{M}^{*} \varrho_{m}^{n}\left(U_{m}\right) \subseteq S\left\|x^{\prime}\right\|_{K}^{*} \varrho_{k}^{n}\left(U_{k}\right)+\varepsilon\left\|x^{\prime}\right\|_{N}^{*} U_{n} .
$$

Here $\left\|x^{\prime}\right\|_{L}^{*}:=\sup _{x \in B_{L}}\left|x^{\prime}(x)\right|$ for $L \leq K$.

Proof. Using the theorem of Palamodov-Retakh we have obtained a first necessary condition for the vanishing of $\operatorname{proj}^{1} E \widetilde{\otimes}_{\alpha} F_{n}$ (Lemma 4.4). As $\operatorname{proj}^{1} \mathcal{X}$ does not change upon replacing $\mathcal{X}$ with an equivalent spectrum, we may assume that all $F_{n}$ satisfy (b2). In particular $\operatorname{ind}_{N} E_{N} \widetilde{\otimes}_{\alpha} F_{n}$ is again regular. So we may assume that the sequence $\left(B_{E_{N} \widetilde{\otimes}_{\alpha} F_{n}}\right)_{N}=$ $\left(\overline{B_{E_{N} \otimes_{\alpha} F_{n}}} E \widetilde{\otimes}_{\alpha} F_{n}\right)_{N}$ is an increasing fundamental sequence of bounded and closed subsets of $E \widetilde{\otimes}_{\alpha} F_{n}$.

In the following we will give the corresponding modifications for replacing $x^{\prime} \in E^{\prime}$ by $x^{\prime} \in E_{K}^{\prime}$ in $\left(S_{2}^{\prime}\right)$, respectively $\left(S_{2}^{\prime}\right)_{0}$. Nothing has to be changed for Lemmas 4.4 and 4.5. Note that $\operatorname{id}_{E} \widetilde{\otimes}_{\alpha} \varrho_{n+1}^{n}\left(B_{n+1, N}\right)$ is contained in a scalar multiple of $B_{n, N}$, which follows from (1). Therefore $\operatorname{id}_{E} \widetilde{\otimes}_{\alpha} \varrho_{k}^{n}\left(B_{k, K}\right)$ is contained in a scalar multiple of $B_{n, K}$ for all $k, n, K$. In the proof of Lemma 4.6 one replaces $x^{\prime} \in E^{\prime} \backslash\{0\}$ by $x^{\prime} \in E_{K}^{\prime} \backslash\{0\}$. By the above, (2) again makes sense. The rest of the proof is analogous. In order to deduce $(Q)$ in the proof of Proposition 4.7, one has to apply $\left(S_{2}^{K \prime}\right)$ to the sequence $\left(i_{K}^{\prime}\left(x_{L}^{\prime}\right)\right)_{L}$ instead of $\left(x_{L}^{\prime}\right)_{L}$. Finally, in (3) one replaces $x^{\prime} \in E^{\prime}$ by $x^{\prime} \in E_{K}^{\prime}$.

In general, we will work with $\left(S_{2}^{K \prime}\right)_{0}$ as a necessary condition for the vanishing of $\operatorname{Tor}_{\alpha}^{1}(E, F)$ but we note that the better-known condition $\left(S_{2}^{*}\right)_{0}$ is also necessary:

Corollary 4.9. If $F$ has a fundamental system of Banach spaces $\left(F_{n}, \varrho_{m}^{n}\right)$ such that $4.1(\mathrm{~b} 2)$ holds, then the condition 
$\left(S_{2}^{*}\right)_{0} \quad$ For all $n$ there is an $N$ and an $m \geq n$ such that for all $M \geq N$, $k \geq m$ and $\varepsilon>0$ we can find $a K \geq M$ and an $S>0$ such that for all $x^{\prime} \in E_{K}^{\prime}$ and $y^{\prime} \in F_{n}^{\prime}$ we have

$$
\left\|x^{\prime}\right\|_{M}^{*}\left\|y^{\prime}\right\|_{m}^{*} \leq \max \left\{S\left\|x^{\prime}\right\|_{K}^{*}\left\|y^{\prime}\right\|_{k}^{*}, \varepsilon\left\|x^{\prime}\right\|_{N}^{*}\left\|y^{\prime}\right\|_{n}^{*}\right\}
$$

is necessary for $\operatorname{Tor}_{\alpha}^{1}(E, F)=0$. Here $\left\|y^{\prime}\right\|_{l}^{*}:=\sup _{y \in U_{l}}\left|y^{\prime}\left(\varrho_{l}^{n}(y)\right)\right|$ for $y^{\prime} \in F_{n}^{\prime}$ and $l \geq n$.

Proof. As the conditions are invariant under replacement with equivalent spectra, we may assume that $\left(F_{n}, \varrho_{m}^{n}\right)$ is reduced. Consequently, the dual spectrum is injective. We consider two cases:

(a) $\left\|x^{\prime}\right\|_{N}^{*} \neq 0$. From $\left(S_{2}^{K \prime}\right)_{0}$ and polarization with respect to $\left\langle F_{n}, F_{n}^{\prime}\right\rangle$, using $(A+B)^{\circ} \supseteq \frac{1}{2}\left(A^{\circ} \cap B^{\circ}\right)$ gives

$$
\frac{1}{2}\left(\frac{1}{S\left\|x^{\prime}\right\|_{K}^{*}}\left(\varrho_{k}^{n}\left(U_{k}\right)\right)^{\circ} \cap \frac{1}{\varepsilon\left\|x^{\prime}\right\|_{N}^{*}} U_{n}^{\circ}\right) \subseteq \frac{1}{\left\|x^{\prime}\right\|_{M}^{*}}\left(\varrho_{m}^{n}\left(U_{m}\right)\right)^{\circ} .
$$

This implies $\left(S_{2}^{*}\right)$, as for $y^{\prime} \in F_{n}^{\prime} \backslash\{0\}$ we have

(i) $y^{\prime} /\left\|y^{\prime}\right\|_{n}^{*} \in U_{n}^{\circ}$, so

(ii) $y^{\prime} /\left\|y^{\prime}\right\|_{k}^{*} \in\left(\varrho_{k}^{n} U_{k}\right)^{\circ}$, so

$$
\frac{1}{2}\left\|\frac{1}{\varepsilon\left\|x^{\prime}\right\|_{N}^{*}} \cdot \frac{y^{\prime}}{\left\|y^{\prime}\right\|_{n}^{*}}\right\|_{m}^{*}\left\|x^{\prime}\right\|_{M}^{*} \leq 1
$$

$$
\frac{1}{2}\left\|\frac{1}{S\left\|x^{\prime}\right\|_{K}^{*}} \cdot \frac{y^{\prime}}{\left\|y^{\prime}\right\|_{k}^{*}}\right\|_{m}^{*}\left\|x^{\prime}\right\|_{M}^{*} \leq 1 .
$$

(b) $\left\|x^{\prime}\right\|_{N}^{*}=0$. The proof is analogous to (a) by omitting the terms with $\left\|x^{\prime}\right\|_{N}^{*}$ and considering the cases $\left\|x^{\prime}\right\|_{M}^{*} \neq 0$ and $\left\|x^{\prime}\right\|_{M}^{*}=0$ separately.

For later purposes it is of interest what information about the pair $(E, F)$ can be deduced from the conditions $\left(S_{2}^{K \prime}\right)$ and $\left(S_{2}^{K \prime}\right)_{0}$, respectively. We will need some further definitions. We call a Fréchet space $F$ countably normed if there is a fundamental system of Banach spaces such that the connecting maps are injective. For instance, a Köthe echelon space $\lambda^{p}(A)$ is countably normed if and only if it has a continuous norm if and only if there is an $N$ with $a_{j, N}>0$ for all $j$. On the other hand, we say that a Fréchet space $F$ is a quojection if $F$ is isomorphic to a projective limit of Banach spaces with surjective connecting maps. The proof of the following lemma is analogous to [26, Lemma 3.2]:

\section{Lemma 4.10. The condition}

$\left(S_{2}^{K \prime}\right) \quad$ For all $n$ there is an $N$ and an $m \geq n$ such that for all $M \geq N$ and $k \geq m$ we can find $a K \geq M$ and an $S>0$ such that for all $x^{\prime} \in E_{K}^{\prime}$ we have

$$
\left\|x^{\prime}\right\|_{M}^{*} \varrho_{m}^{n}\left(U_{m}\right) \subseteq S\left(\left\|x^{\prime}\right\|_{K}^{*} \varrho_{k}^{n}\left(U_{k}\right)+\left\|x^{\prime}\right\|_{N}^{*} U_{n}\right)
$$

implies that either $E_{b}^{\prime}$ is countably normed or $F$ is a quojection. 
Now let us turn to sufficient conditions for the vanishing of $\operatorname{Tor}_{\alpha}^{1}(E, F)$.

REMARK 4.11. Clearly $\left(S_{2}^{K \prime}\right)_{0}$ implies

$\left(S_{3}^{K \prime}\right)_{0} \quad$ For all $n$ there is an $m \geq n$ such that for all $k \geq m$ we can find an $N$ such that for all $M \geq N$ and $\varepsilon>0$ there is a $K \geq M$ and an $S>0$ with

$$
\left\|x^{\prime}\right\|_{M}^{*} \varrho_{m}^{n}\left(U_{m}\right) \subseteq S\left\|x^{\prime}\right\|_{K}^{*} \varrho_{k}^{n}\left(U_{k}\right)+\varepsilon\left\|x^{\prime}\right\|_{N}^{*} U_{n}
$$

for all $x^{\prime} \in E_{K}^{\prime}$.

The general strategy for showing that $\left(S_{3}^{K^{\prime}}\right)_{0}$ is also sufficient for the vanishing of $\operatorname{Tor}_{\alpha}^{1}(E, F)$ in special cases is roughly the following: Using information about the spaces $E$ and/or $F$ we will deduce further conditions from $\left(S_{3}^{K \prime}\right)_{0}$ by polarization in order to decompose elementary tensors. To be more precise, we wish to show that

$\left(S_{3}^{\otimes}\right)_{0} \quad$ For all $n$ there is an $m \geq n$ such that for all $k \geq m$ we can find an $N$ such that for all $M \geq N$ and $\varepsilon>0$ there is a $K \geq M$ and an $S>0$ with

$$
\operatorname{id}_{E} \widetilde{\otimes}_{\alpha} \varrho_{m}^{n}\left(B_{E_{M}} \otimes B_{F_{m}}\right) \subseteq S \operatorname{id}_{E} \widetilde{\otimes}_{\alpha} \varrho_{k}^{n}\left(B_{E_{K} \widetilde{\otimes}_{\alpha} F_{k}}\right)+\varepsilon B_{E_{N} \widetilde{\otimes}_{\alpha} F_{n}}
$$

is necessary for $\left(S_{3}^{K \prime}\right)_{0}$. Another condition in this context is

$\left(S_{3}^{\widetilde{\otimes}}\right)_{0} \quad$ For all $n$ there is an $m \geq n$ such that for all $k \geq m$ we can find an $N$ such that for all $M \geq N$ and $\varepsilon>0$ there is an $K \geq M$ and an $S>0$ with

$$
\operatorname{id}_{E} \widetilde{\otimes}_{\alpha} \varrho_{m}^{n}\left(B_{E_{M} \widetilde{\otimes}_{\alpha} F_{m}}\right) \subseteq S \operatorname{id}_{E} \widetilde{\otimes}_{\alpha} \varrho_{k}^{n}\left(B_{E_{K} \widetilde{\otimes}_{\alpha} F_{k}}\right)+\varepsilon B_{E_{N} \widetilde{\otimes}_{\alpha} F_{n}} .
$$

LEMMA 4.12. For the projective tensor product, $\left(S_{3}^{\otimes}\right)_{0}$ always implies $\left(S_{3}^{\widetilde{\otimes}}\right)_{0}$. If $F$ has a fundamental system of Banach spaces $\left(F_{n}, \varrho_{m}^{n}\right)$ which satisfies Setting 4.1(b2), we even get

$\left(S_{3}\right)_{0} \quad$ For all $n$ there is an $m \geq n$ such that for all $k \geq m$ there is an $N$ such that for all $M \geq N$ and $\varepsilon>0$ there is a $K \geq M$ and an $S>0$ with

$$
\operatorname{id}_{E} \widetilde{\otimes}_{\pi} \varrho_{m}^{n}\left(B_{m, M}\right) \subseteq S \operatorname{id}_{E} \widetilde{\otimes}_{\pi} \varrho_{k}^{n}\left(B_{k, K}\right)+\varepsilon B_{n, N} .
$$

Proof. We first remark that for all $z \in B_{E_{M} \widetilde{\otimes}_{\pi} F_{m}}$ there are sequences $\left(\lambda_{j}\right)_{j} \in l_{1}$ with $\left\|\left(\lambda_{j}\right)_{j}\right\|_{1} \leq 2,\left(x_{j}\right)_{j} \in B_{E_{M}}$ and $\left(y_{j}\right)_{j} \in B_{F_{m}}$ such that $z$ has a representation $z=\sum_{j} \lambda_{j}\left(x_{j} \otimes y_{j}\right)$ : By definition of the projective norm there is a representation $z=\sum_{j=1}^{\infty} \widetilde{x}_{j} \otimes \widetilde{y}_{j}$ with $\sum_{j=1}^{\infty}\left\|\widetilde{x}_{j}\right\| \cdot\left\|\widetilde{y}_{j}\right\| \leq 2$. Setting $\lambda_{j}:=\left\|\widetilde{x}_{j}\right\| \cdot\left\|\widetilde{y}_{j}\right\|, x_{j}:=\widetilde{x}_{j} /\left\|\widetilde{x}_{j}\right\|$ and $y_{j}:=\widetilde{y}_{j} /\left\|\widetilde{y}_{j}\right\|$ yields the assertion, by cancelling zero summands. 
Now for $z=\sum_{j} \lambda_{j}\left(x_{j} \otimes y_{j}\right) \in B_{E_{M} \widetilde{\otimes}_{\pi} F_{m}}$ as above, from $\left(S_{2}^{\otimes}\right)_{0}$, we get

$$
\operatorname{id}_{E} \widetilde{\otimes}_{\pi} \varrho_{m}^{n}(z)=\sum_{j} \lambda_{j}\left(S \operatorname{id}_{E} \widetilde{\otimes}_{\pi} \varrho_{k}^{n}\left(w_{j}\right)+\varepsilon v_{j}\right)
$$

with $\left(w_{j}\right)_{j} \in B_{E_{K} \widetilde{\otimes}_{\pi} F_{k}}^{\mathbb{N}}$ and $\left(v_{j}\right)_{j} \in B_{E_{N} \widetilde{\otimes}_{\pi} F_{n}}^{\mathbb{N}}$. As $\left(\lambda_{j}\right)_{j} \in l_{1}$ and the sequences $\left(w_{j}\right)_{j},\left(v_{j}\right)_{j}$ are bounded, the sums

$$
\sum_{j} \lambda_{j} w_{j} \in 2 B_{E_{K} \tilde{\otimes}_{\pi} F_{k}} \text { and } \quad \sum_{j} \lambda_{j} v_{j} \in 2 B_{E_{N} \widetilde{\otimes}_{\pi} F_{n}}
$$

converge. We conclude

$$
\begin{aligned}
\operatorname{id}_{E} \widetilde{\otimes}_{\pi} \varrho_{m}^{n}(z) & =S \operatorname{id}_{E} \widetilde{\otimes}_{\pi} \varrho_{k}^{n}\left(\sum_{j} \lambda_{j} w_{j}\right)+\varepsilon \sum_{j} \lambda_{j} v_{j} \\
& \in 2 S \operatorname{id}_{E} \widetilde{\otimes}_{\pi} \varrho_{k}^{n}\left(B_{E_{K} \widetilde{\otimes}_{\pi} F_{k}}\right)+2 \varepsilon B_{E_{N} \widetilde{\otimes}_{\pi} F_{n}} .
\end{aligned}
$$

From this we get the first assertion. Setting 4.1(b2) says that we may assume that $B_{m, M}=C_{m, M} B_{E_{M} \widetilde{\otimes}_{\pi} F_{m}}$, which completes the proof.

Let us summarize our results:

THEOREM 4.13. The condition $\left(S_{3}\right)_{0}$ is sufficient for the vanishing of $\operatorname{proj}^{1} E \widetilde{\otimes}_{\alpha} F_{n}$. If furthermore $F$ is locally $E \widetilde{\otimes}_{\alpha} \cdot$-acyclic we have $0=$ $\operatorname{proj}^{1} E \widetilde{\otimes}_{\alpha} F_{n}=\operatorname{Tor}_{\alpha}^{1}(E, F)$. In the case of $\alpha=\pi$ one may replace $\left(S_{3}\right)_{0}$ by $\left(S_{3}^{\otimes}\right)_{0}$, provided that the interchangeability property of Setting 4.1(b2) holds.

Proof. The assertion follows from [31, Theorem 3.2.14], Corollary 2.13 and Lemma 4.12.

5. The standard cases. In analogy to Ext-theory (see [26] and [4]), we will consider four so-called standard cases for Tor-theory, namely:

(I) $F=\lambda^{\infty}(B), \alpha=\varepsilon$,

(II) $E$ a nuclear (DF)-space, $\alpha$ an arbitrary tensor norm,

(III) $F$ a nuclear Fréchet space, $\alpha$ an arbitrary tensor norm,

(IV) $E=k^{1}(A)$ a coechelon space of type one and $\alpha=\pi$, or $E=k^{0}(A)$ a coechelon space of type zero and $\alpha=\varepsilon$.

First we present a result concerning the injective tensor product, which can be deduced from Ext-theory and is based on an easy argument using equivalent functors. It is well-known that for (DFM)-spaces $E$ with the a.p. the functors $E \widetilde{\otimes}_{\varepsilon} \cdot$ and $L\left(E_{b}^{\prime}, \cdot\right): \mathcal{F S} \rightarrow \mathcal{L S}$ are naturally equivalent. As a consequence we obtain

Theorem 5.1. Let $E$ be a (DFM)-space with the a.p. and $F$ a Fréchet space. Then:

(i) $\operatorname{Tor}_{\varepsilon}^{l}(E, F)=\operatorname{Ext}^{l}\left(E_{b}^{\prime}, F\right)$ for all $l \geq 0$.

(ii) $E \widetilde{\otimes}_{\varepsilon} \cdot: \mathcal{F S} \rightarrow \mathcal{L S}$ is left exact, in particular $\operatorname{Tor}_{\varepsilon}^{0}(E, F)=E \widetilde{\otimes}_{\varepsilon} F$. 
(iii) $\operatorname{Tor}_{\varepsilon}^{1}(E, F)=0$ if and only if for all exact $\mathcal{F} \mathcal{S}$-sequences $0 \rightarrow F \rightarrow$ $G \rightarrow H \rightarrow 0$ the $E \widetilde{\otimes}_{\varepsilon} \cdot$-tensored sequence $0 \rightarrow E \widetilde{\otimes}_{\varepsilon} F \rightarrow E \widetilde{\otimes}_{\varepsilon} G \rightarrow$ $E \widetilde{\otimes}_{\varepsilon} H \rightarrow 0$ is (topologically) exact.

(iv) $\operatorname{Tor}_{\varepsilon}^{l}(E, F)=0$ for quasinormable $F=\lambda^{\infty}(B)$ and $l \geq 2$.

(v) If $E$ is proper and $F=\lambda^{\infty}(B)$, then $\operatorname{Tor}_{\varepsilon}^{1}(E, F)=0$ if and only if the condition $\left(S_{3}^{K \prime}\right)_{0}$ from Remark 4.11 holds.

Proof. As $E \widetilde{\otimes}_{\varepsilon} \cdot \simeq L\left(E_{b}^{\prime}, \cdot\right): \mathcal{F S} \rightarrow \mathcal{L S}$, their derivatives are also equivalent (up to natural isomorphism). This implies (i) and (ii). For (iii) one uses Remark 2.2, and for (iv) the fact that quasinormable Köthe spaces of type infinity are locally injective (see [26]). If $E$ is a proper (DFM)-space, then its dual space $E_{b}^{\prime}$ is a proper Fréchet space and we get (iv) from an analysis of [26, 4] and [13] (cf. also [31, Section 5.2]).

REMARK 5.2. Note that in case (v), the assumption that $E$ be a (DFM)space with the a.p. can be relinquished for appropriate $F$, such as nuclear Fréchet spaces. On the other hand, for $F=\lambda^{\infty}(B)$ we remark the following: Let $E=\operatorname{ind}_{N} E_{N}$ be a compact regular (LB)-space and $F$ quasinormable (note [26, Remark p. 167] and Example 4.3(2)). Now the identity $E_{M} \widetilde{\otimes}_{\varepsilon} F_{m}=E_{M} \widetilde{\otimes}_{\varepsilon}\left(l_{1}\left(b_{j, m}^{-1}\right)\right)_{b}^{\prime}=\mathcal{K}\left(l_{1}\left(b_{j, m}^{-1}\right), E_{M}\right)$ leads to the problem of decomposing compact operators into sums of suitable compact operators. It is not clear whether this is possible.

Next let us consider the case where $E$ is a nuclear (DF)-space. This is treated very similarly to the above, using arguments about equivalent functors:

REMARK 5.3. By a result of A. Grothendieck a locally convex space $E$ is nuclear if and only if the functors $E \widetilde{\otimes}_{\pi} \cdot$ and $E \widetilde{\otimes}_{\varepsilon} \cdot \mathcal{F} \mathcal{S} \rightarrow \mathcal{L S}$ coincide. In that case, all tensor norms $\alpha$ agree.

Now we can formulate and prove the main result concerning the case of nuclear (DF)-spaces:

TheOREM 5.4. For a nuclear $(D F)$-space $E$, a Fréchet space $F$ with a fundamental system of Banach spaces $\left(F_{n}, \varrho_{m}^{n}\right)$ and a tensor norm $\alpha$, one has:

(i) $\operatorname{Tor}_{\alpha}^{l}(E, F)=\operatorname{Ext}^{l}\left(E_{b}^{\prime}, F\right)$ for all $l \geq 0$.

(ii) $E \widetilde{\otimes}_{\alpha} \cdot: \mathcal{F S} \rightarrow \mathcal{L S}$ is left exact, in particular $\operatorname{Tor}_{\alpha}^{0}(E, F)=E \widetilde{\otimes}_{\alpha} F$.

(iii) $\operatorname{Tor}_{\alpha}^{1}(E, F)=0$ if and only if for any short exact $\mathcal{F} \mathcal{S}$-sequence the $E \widetilde{\otimes}_{\alpha} \cdot-$ tensored sequence is (topologically) exact.

(iv) $\operatorname{Tor}_{\alpha}^{l}(E, F)=0$ for $l \geq 2$.

(v) If $E$ is proper, then $\operatorname{Tor}_{\alpha}^{1}(E, F)=0$ if and only if the condition $\left(S_{3}^{K \prime}\right)_{0}$ is satisfied. 
Proof. By nuclearity we may assume that $\alpha=\varepsilon$. It is well-known that nuclear spaces have the a.p. and that the strong duals of nuclear (DF)-spaces are nuclear Fréchet spaces.

First let us prove the assertion for complete spaces $E$. In this case, $E$ is a (DFM)-space with the a.p., so the functors $E \widetilde{\otimes}_{\varepsilon} \cdot$ and $L\left(E_{b}^{\prime}, \cdot\right)$ are naturally equivalent. From this we get all the assertions as in the proof of Theorem 5.1. For (ii) one may also use Corollary 1.8(a), and for (iv) the fact that every Banach space is $E \widetilde{\otimes}_{\alpha}$-acyclic (this follows for instance from $E_{b}^{\prime}$ being locally projective).

It is well-known that for a nuclear (DF)-space $E$ the completion $\widetilde{E}$ is again a nuclear $(\mathrm{DF})$-space and that $(\mathrm{DF})$-spaces are large, so $b\left(\widetilde{E}^{\prime}, \widetilde{E}\right)=$ $b\left(E^{\prime}, E\right)$. Summarizing, we have $E \widetilde{\otimes}_{\varepsilon} \cdot \simeq \widetilde{E} \widetilde{\otimes}_{\varepsilon} \cdot \simeq L\left(\widetilde{E}_{b}^{\prime}, \cdot\right) \simeq L\left(E_{b}^{\prime}, \cdot\right)$, which completes the proof.

Now let us consider the nuclear case in the Fréchet variable: For a locally convex space $E$, a nuclear Fréchet space $F$ and a tensor norm $\alpha$, the condition $\operatorname{Tor}_{\alpha}^{1}(E, F)=0$ does not depend on the tensor norm: Let $\left(F_{n}, \varrho_{m}^{n}\right)$ be a fundamental system of Banach spaces for $F$ such that the connecting maps $\varrho_{n+1}^{n}: F_{n+1} \rightarrow F_{n}$ are nuclear. By [10, Proposition 17.3.8] the map $\operatorname{id}_{E} \widetilde{\otimes} \varrho_{n+1}^{n}: E \widetilde{\otimes}_{\alpha} F_{n+1} \rightarrow E \widetilde{\otimes}_{\pi} F_{n}$ is continuous. An easy computation with elementary tensors shows that the diagram

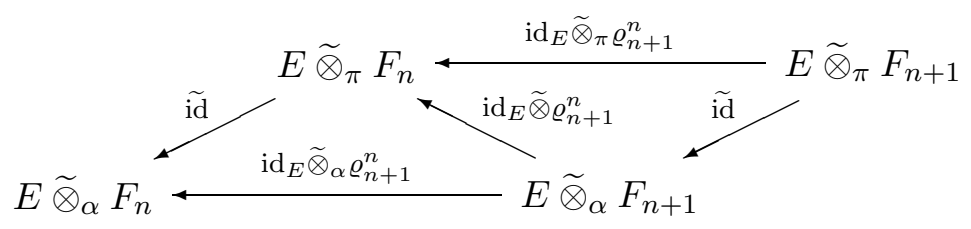

commutes. Hence the projective spectra $\left(E \widetilde{\otimes}_{\alpha} F_{n}, \operatorname{id}_{E} \widetilde{\otimes}_{\alpha} \varrho_{m}^{n}\right)$ and $\left(E \widetilde{\otimes}_{\pi} F_{n}\right.$, $\left.\operatorname{id}_{E} \widetilde{\otimes}_{\pi} \varrho_{m}^{n}\right)$ are equivalent and by Example 2.5 we have $\operatorname{proj}^{1} E \widetilde{\otimes}_{\alpha} F_{n}=$ $\operatorname{proj}^{1} E \widetilde{\otimes}_{\pi} F_{n}$. As $F$ is nuclear, $F$ has a fundamental system of injective Banach spaces, and Corollary 2.13 implies:

Proposition 5.5. For a tensor norm $\alpha$, a locally convex space $E$ and a nuclear Fréchet space $F$ with a fundamental system of Banach spaces $\left(F_{n}, \varrho_{m}^{n}\right)$ the following holds for $l \geq 1$ :

$$
\operatorname{Tor}_{\alpha}^{l}(E, F)=\operatorname{proj}^{l} E \widetilde{\otimes}_{\alpha} F_{n}=\operatorname{proj}^{l} E \widetilde{\otimes}_{\pi} F_{n}=\operatorname{Tor}_{\pi}^{l}(E, F) .
$$

With a similar argument we prove:

REMARK 5.6. Let $\alpha$ be a tensor norm, $H$ a distinguished Fréchet space and $F$ a nuclear Fréchet space. Then $\operatorname{Tor}_{\alpha}^{1}\left(H_{b}^{\prime}, F\right)=\operatorname{Ext}^{1}(H, F)$.

Proof. By Proposition 5.5 we may assume that $\alpha=\pi$. Let $\|\cdot\|_{1} \leq$ $\|\cdot\|_{2} \leq \cdots$ be a fundamental sequence of seminorms of $H$ and $\left(F_{n}, \varrho_{m}^{n}\right)$ a fundamental system of $l_{1}$-spaces for $F$ with nuclear connecting maps. Then 
the map

$$
L\left(H, F_{n+1}\right)=\operatorname{ind}_{N} L\left(H_{N}, F_{n+1}\right) \stackrel{\left(\varrho_{n+1}^{n}\right)_{*}}{\longrightarrow} L\left(H, F_{n}\right)=\operatorname{ind}_{N} L\left(H_{N}, F_{n}\right)
$$

factors through $\operatorname{ind}_{N} \mathcal{N}\left(H_{N}, F_{n}\right)=\operatorname{ind}_{N}\left(H_{N}^{\prime} \widetilde{\otimes}_{\pi} F_{n}\right)=H_{b}^{\prime} \widetilde{\otimes}_{\pi} F_{n}$ (Example $4.3(1))$ and the diagram

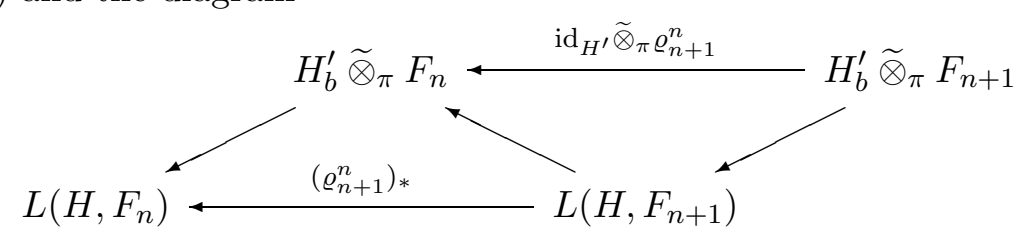

commutes. Here $\mathcal{N}(X, Y)$ denotes the space of nuclear maps from $X$ to $Y$, where $X$ and $Y$ are Banach spaces. Consequently, $\operatorname{Tor}_{\pi}^{1}\left(H_{b}^{\prime}, F\right)=$ $\operatorname{proj}^{1} H_{b}^{\prime} \widetilde{\otimes}_{\pi} F_{n}=\operatorname{proj}^{1} L\left(H, F_{n}\right)=\operatorname{Ext}^{1}(H, F)$.

Let us return to the general case; we may assume that $\alpha=\pi$. Further let $E=\operatorname{ind}_{N} E_{N}$ be a proper, complete (LB)-space, $B_{1}=B_{E_{1}} \subseteq B_{2}=$ $B_{E_{2}} \subseteq \cdots$ a fundamental sequence of closed and bounded subsets of $E$, and $F$ a nuclear Fréchet space with a fundamental system of Banach spaces $\left(F_{n}, \varrho_{m}^{n}\right), U_{n}:=B_{F_{n}}$.

In the last section we showed that the condition $\left(S_{3}^{K \prime}\right)_{0}$ is necessary for $\operatorname{Tor}_{\pi}^{1}(E, F)=0$ (use Theorem 4.8). The next key result is:

Proposition 5.7. In the above notation, the condition $\left(S_{3}^{K \prime}\right)_{0}$ implies $\operatorname{proj}^{1} E \widetilde{\otimes}_{\pi} F_{n}=0$.

Proof. We may assume that $\left(F_{n}, \varrho_{m}^{n}\right)$ is reduced (as the conditions are invariant with respect to equivalent projective spectra). By Example 4.3(i) and Theorem 4.13 it suffices to show that the following condition holds:

$\left(S_{3}^{\otimes}\right)_{0} \quad$ For all $n$ there is an $m \geq n$ such that for all $k \geq m$ we can find an $N$ such that for all $M \geq N$ and $\varepsilon>0$ there is a $K \geq M$ and an $S>0$ with

$$
\operatorname{id}_{E} \widetilde{\otimes}_{\pi} \varrho_{m}^{n}\left(B_{E_{M}} \otimes B_{F_{m}}\right) \subseteq \operatorname{Sid}_{E} \widetilde{\otimes}_{\pi} \varrho_{k}^{n}\left(B_{E_{K} \widetilde{\otimes}_{\pi} F_{k}}\right)+\varepsilon B_{E_{N} \widetilde{\otimes}_{\pi} F_{n}} .
$$

From $\left(S_{3}^{K \prime}\right)_{0}$ we first deduce the condition

(Pol) For all $n$ there is an $m \geq n$ such that for all $k \geq m$ we can find an $N$ such that for all $M \geq N$ and $\varepsilon>0$ there is a $K \geq M$ and an $S>0$ with

$$
\left\|y^{\prime}\right\|_{m}^{*} B_{M} \subseteq S\left\|y^{\prime}\right\|_{k}^{*} B_{K}+\varepsilon\left\|y^{\prime}\right\|_{n}^{*} B_{N}
$$

for all $y^{\prime} \in F_{n}^{\prime}$, where $\left\|y^{\prime}\right\|_{l}^{*}:=\sup _{y \in U_{l}}\left|y^{\prime}\left(\varrho_{l}^{n}(y)\right)\right|$ for $y^{\prime} \in F_{n}^{\prime}$ and $l \geq n$.

In analogy to Corollary 4.9, the corresponding condition 
$\left(S_{3}^{*}\right)_{0} \quad$ For all $n$ there is an $m \geq n$ such that for all $k \geq m$ we can find an $N$ such that for all $M \geq N$ and $\varepsilon>0$ there is a $K \geq M$ and an $S>0$ with

$$
\left\|x^{\prime}\right\|_{M}^{*}\left\|y^{\prime}\right\|_{m}^{*} \leq \max \left\{S\left\|x^{\prime}\right\|_{K}^{*}\left\|y^{\prime}\right\|_{k}^{*}, \varepsilon\left\|x^{\prime}\right\|_{N}^{*}\left\|y^{\prime}\right\|_{n}^{*}\right\}
$$

for all $x^{\prime} \in E_{K}^{\prime}$ and $y^{\prime} \in F_{n}^{\prime}$

is necessary for $\left(S_{3}^{K \prime}\right)_{0}$ to hold. Let $y^{\prime} \in F_{n}^{\prime} \backslash\{0\}$, so $\left\|y^{\prime}\right\|_{j}^{*} \neq 0$ for $j=n, m, k$. In what follows we polarize with respect to the dual system $\left\langle E_{K}, E_{K}^{\prime}\right\rangle$. The condition $\left(S_{3}^{*}\right)_{0}$ gives

$$
\frac{1}{S\left\|y^{\prime}\right\|_{k}^{*}} B_{K}^{\circ} \cap \frac{1}{\varepsilon\left\|y^{\prime}\right\|_{n}^{*}} B_{N}^{\circ} \subseteq \frac{1}{\left\|y^{\prime}\right\|_{m}^{*}} B_{M}^{\circ} .
$$

With $\left(A^{\circ \circ} \cap B^{\circ \circ}\right)^{\circ} \subseteq \overline{A^{\circ \circ}+B^{\circ \circ}}$ the bipolar theorem yields

$$
\begin{aligned}
\left\|y^{\prime}\right\|_{m}^{*} B_{M} & \subseteq\left\|y^{\prime}\right\|_{m}^{*}\left(B_{M}\right)^{\circ \circ} \subseteq \overline{S\left\|y^{\prime}\right\|_{k}^{*} B_{K}+\varepsilon\left\|y^{\prime}\right\|_{n}^{*} \overline{B_{N}} E_{K}} E_{K} \\
& \subseteq 2 S\left\|y^{\prime}\right\|_{k}^{*} B_{K}+\varepsilon\left\|y^{\prime}\right\|_{n}^{*} \overline{B_{N}} E_{K} \\
& \subseteq 2 S\left\|y^{\prime}\right\|_{k}^{*} B_{K}+\varepsilon\left\|y^{\prime}\right\|_{n}^{*}\left(B_{N}+\frac{S}{\varepsilon} \frac{\left\|y^{\prime}\right\|_{k}^{*}}{\left\|y^{\prime}\right\|_{n}^{*}} B_{K}\right) \\
& \subseteq 3 S\left\|y^{\prime}\right\|_{k}^{*} B_{K}+\varepsilon\left\|y^{\prime}\right\|_{n}^{*} B_{N} .
\end{aligned}
$$

The implication $\left(S_{3}^{K \prime}\right)_{0} \Rightarrow(\mathrm{Pol})$ follows. We will proceed with the proof after

REMARK 5.8. If one considers the condition $\left(S_{3}^{\prime}\right)_{0}$ (see Lemma 4.7) instead of $\left(S_{3}^{K \prime}\right)_{0}$ one has to polarize with respect to the dual system $\left\langle E, E^{\prime}\right\rangle$ and obtains

$$
\left\|y^{\prime}\right\|_{m}^{*} B_{M} \subseteq \overline{S\left\|y^{\prime}\right\|_{k}^{*} B_{K}+\varepsilon\left\|y^{\prime}\right\|_{n}^{*} B_{N}}{ }^{E} .
$$

In order to eliminate the closure operation one may require for instance $E$ to be a dual space or a retractive (LB)-space, i.e., an (LB)-space such that for all bounded subsets $B \subseteq E$ there is an $L$ with $B \subseteq E_{L}$ and $\left.\mathcal{T}_{E}\right|_{B}=\left.\mathcal{T}_{L}\right|_{B}$ (set $\left.B:=S\left\|y^{\prime}\right\|_{k}^{*} B_{K}+\varepsilon\left\|y^{\prime}\right\|_{n}^{*} B_{N}\right)$.

As $F$ is nuclear, $F$ has a reduced projective spectrum $\left(F_{n}, \varrho_{m}^{n}\right)$ consisting of Hilbert spaces and nuclear connecting maps $\varrho_{n+1}^{n}$. Let $\langle\cdot, \cdot\rangle_{n}$ denote the inner product on $F_{n}$.

In what follows, we always assume that $k \geq m \geq n+2$. As $\varrho_{k+1}^{n+1}$ : $F_{k+1} \rightarrow F_{n+1}$ is nuclear, there is an orthonormal system $\left(e_{j}\right)_{j}$ in $F_{k+1}$, an orthonormal basis $\left(f_{j}\right)_{j}$ in $F_{n+1}$ and a sequence $\left(a_{j}\right)_{j}$ of positive numbers such that for all $y \in F_{k+1}$ we have the representation

$$
\varrho_{k+1}^{n+1}(y)=\sum_{j} a_{j}\left\langle y, e_{j}\right\rangle_{k+1} f_{j} .
$$


In order to verify the condition $\left(S_{3}^{\otimes}\right)_{0}$ we need some general estimates related to the nuclear maps $\varrho_{\nu+1}^{\nu}$ (see [26]); these are in particular Hilbert-Schmidt operators. Below, $\nu_{1}$ denotes the nuclear and $\nu_{2}$ the Hilbert-Schmidt norm. For $\nu \in \mathbb{N}$ and any orthonormal system $\left(g_{j}\right)_{j}$ in $F_{\nu+1}$ we get, for all $x \in F_{\nu+1}$ (using the estimates of Hölder and Bessel),

$$
\sum_{j}\left|\left\langle x, g_{j}\right\rangle_{\nu+1}\right| \cdot\left\|\varrho_{\nu+1}^{\nu}\left(g_{j}\right)\right\|_{\nu} \leq \nu_{2}\left(\varrho_{\nu+1}^{\nu}\right)\|x\|_{\nu+1}
$$

Consequently, $\delta_{\nu+1}: F_{\nu+1} \rightarrow l_{1}, x \mapsto\left(\left\langle x, g_{j}\right\rangle_{\nu+1}\left\|\varrho_{\nu+1}^{\nu}\left(g_{j}\right)\right\|_{\nu}\right)_{j}$, is a continuous linear operator and therefore $\delta_{\nu+1} \circ \varrho_{\nu+2}^{\nu+1}$ is again nuclear. As we have an isometric isomorphism $\mathcal{N}\left(F_{\nu+2}, l_{1}\right)=l_{1} \widetilde{\otimes}_{\pi} F_{\nu+2}^{\prime}=l_{1}\left(F_{\nu+2}^{\prime}\right)$, it follows that $\nu_{1}\left(\delta_{\nu+1} \circ \varrho_{\nu+2}^{\nu+1}\right)$ equals the $l_{1}$-sum of the sequence

$$
\left(\sup _{\|x\|_{\nu+2} \leq 1}\left|\left\langle\varrho_{\nu+2}^{\nu+1}(x), g_{j}\right\rangle_{\nu+1}\right| \cdot\left\|\varrho_{\nu+1}^{\nu}\left(g_{j}\right)\right\|_{\nu}\right)_{j}
$$

Thus there is a positive sequence $\left(\lambda_{j}^{(\nu)}\right)_{j} \in l_{1}$ with

$$
\begin{aligned}
\sum_{j} \lambda_{j}^{(\nu)} & \leq 2 \nu_{1}\left(\delta_{\nu+1} \circ \varrho_{\nu+2}^{\nu+1}\right) \leq 2\left\|\delta_{\nu+1}\right\| \nu_{1}\left(\varrho_{\nu+2}^{\nu+1}\right) \\
& \leq 2 \nu_{2}\left(\varrho_{\nu+1}^{\nu}\right) \nu_{1}\left(\varrho_{\nu+2}^{\nu+1}\right)=: L(\nu)
\end{aligned}
$$

and

$$
\sup _{\|x\|_{\nu+2} \leq 1}\left|\left\langle\varrho_{\nu+2}^{\nu+1}(x), g_{j}\right\rangle_{\nu+1}\right| \cdot\left\|\varrho_{\nu+1}^{\nu}\left(g_{j}\right)\right\|_{\nu} \leq \lambda_{j}^{(\nu)} \quad \text { for all } j .
$$

Let $\gamma_{j}^{(\nu)}:=\left(\lambda_{j}^{(\nu)}\right)^{-1}\left\|\varrho_{\nu+1}^{\nu}\left(g_{j}\right)\right\|_{\nu}$. By (5) we get, for all $x \in F_{\nu+2}$,

$$
\sup _{j} \gamma_{j}^{(\nu)}\left|\left\langle\varrho_{\nu+2}^{\nu+1}(x), g_{j}\right\rangle_{\nu+1}\right| \leq\|x\|_{\nu+2} \text {. }
$$

With $y_{j}^{\prime} \in F_{n+1}^{\prime}, y_{j}^{\prime}(y):=\left\langle y, f_{j}\right\rangle_{n+1}$, and using the nuclear representations of $\varrho_{k+1}^{n+1}$ we have for all $x \in F_{k+2}$ the identity

$$
y_{j}^{\prime}\left(\varrho_{k+2}^{n+1}(x)\right)=\left\langle\varrho_{k+1}^{n+1}\left(\varrho_{k+2}^{k+1}(x)\right), f_{j}\right\rangle_{n+1}=a_{j}\left\langle\varrho_{k+2}^{k+1}(x), e_{j}\right\rangle_{k+1} .
$$

Setting $\nu=k$ and $g_{j}=e_{j}$ in (6) gives

$$
\sup _{j} \gamma_{j}^{(k)} a_{j}^{-1}\left\|y_{j}^{\prime}\right\|_{k+2}^{*}=\sup _{j} \sup _{\|x\|_{k+2} \leq 1} \gamma_{j}^{(k)}\left|\left\langle\varrho_{k+2}^{k+1}(x), e_{j}\right\rangle_{k+1}\right| \leq 1 .
$$

Setting $\nu=n$ and $g_{j}=f_{j}$ in (6) gives in an analogous manner

$$
\sup _{j} \gamma_{j}^{(n)}\left\|y_{j}^{\prime}\right\|_{n+2}^{*} \leq 1
$$

We now return to the proof of $\left(S_{3}^{\otimes}\right)_{0}$. Let $A \in B_{E_{M}} \otimes B_{F_{m}}$ be given. For $j \in \mathbb{N}$ we consider the continuous linear maps $\varphi_{j}: E_{M} \otimes_{\pi} F_{m} \rightarrow E_{M}$, 
$x \otimes y \mapsto\left\langle\varrho_{m}^{n+1}(y), f_{j}\right\rangle_{n+1} x$, and set $A_{j}:=\varphi_{j}(A) \in E_{M}$. Then for $x^{\prime} \in E^{\prime}$ with $\left\|x^{\prime}\right\|_{M}^{*} \leq 1$ we conclude that

$$
\begin{aligned}
\left|x^{\prime}\left(A_{j}\right)\right| & =\left|x^{\prime}\left(\varphi_{j}(A)\right)\right| \leq\left\|\varphi_{j}(A)\right\|_{E_{M}} \leq\left\|\varphi_{j}\right\|=\sup _{z \in B_{E_{M} \otimes_{\pi} F_{m}}}\left\|\varphi_{j}(z)\right\|_{E_{M}} \\
& \leq\left\|y_{j}^{\prime}\right\|_{m}^{*} .
\end{aligned}
$$

Consequently, $A_{j} \in\left\|y_{j}^{\prime}\right\|_{m}^{*} B_{M}$. Now let $n$ in $\left(S_{3}^{\otimes}\right)_{0}$ be given. Applying (Pol) to $n+2$ we find an $m \geq n+2$ so that for given $k \geq m$ in $\left(S_{3}^{\otimes}\right)_{0}$ resp. for $k+2 \geq m$ in (Pol) there is an $N$ such that for all $M \geq N$ and $\varepsilon>0$ in $\left(S_{3}^{\otimes}\right)_{0}$ resp. $M \geq N$ and $\widetilde{\varepsilon}:=\varepsilon / L(n)$ in $(\mathrm{Pol})$ there are $K \geq M$ and $\widetilde{S}>0$ such that the above $A_{j}$ can be written as $A_{j}=B_{j}+C_{j}$, where $B_{j} \in \widetilde{S}\left\|y_{j}^{\prime}\right\|_{k+2}^{*} B_{K}$ and $C_{j} \in \widetilde{\varepsilon}\left\|y_{j}^{\prime}\right\|_{n+2}^{*} B_{N}$.

Now define $B \in E_{K} \widetilde{\otimes}_{\pi} F_{k}$ and $C \in E_{N} \widetilde{\otimes}_{\pi} F_{n}$ by

$$
B:=\frac{1}{L(k) \widetilde{S}} \sum_{j} a_{j}^{-1} B_{j} \otimes \varrho_{k+1}^{k}\left(e_{j}\right), \quad C:=\frac{1}{L(n) \widetilde{\varepsilon}} \sum_{j} C_{j} \otimes \varrho_{n+1}^{n}\left(f_{j}\right) .
$$

From the estimate

$$
\begin{aligned}
\pi(B) & \leq \frac{1}{L(k) \widetilde{S}} \sum_{j} a_{j}^{-1}\left\|B_{j}\right\|_{E_{K}}\left\|\varrho_{k+1}^{k}\left(e_{j}\right)\right\|_{k} \\
& \leq \frac{1}{L(k) \widetilde{S}} \sum_{j} a_{j}^{-1} \widetilde{S}\left\|y_{j}^{\prime}\right\|_{k+2}^{*}\left\|\varrho_{k+1}^{k}\left(e_{j}\right)\right\|_{k} \\
& \stackrel{(4)}{\leq} \sup _{j} a_{j}^{-1}\left\|y_{j}^{\prime}\right\|_{k+2}^{*} \gamma_{j}^{(k)} \stackrel{(7)}{\leq} 1
\end{aligned}
$$

we obtain absolute convergence of the sum $B$ in $E_{K} \widetilde{\otimes}_{\pi} F_{k}$ (and therefore in $E \widetilde{\otimes}_{\pi} F_{k}$ ), and $B \in B_{E_{K} \widetilde{\otimes}_{\pi} F_{k}}$. Similarly we have

$$
\begin{aligned}
\pi(C) & \leq \frac{1}{L(n) \widetilde{\varepsilon}} \sum_{j}\left\|C_{j}\right\|_{E_{N}}\left\|\varrho_{n+1}^{n}\left(f_{j}\right)\right\|_{n} \\
& \leq \frac{1}{L(n) \widetilde{\varepsilon}} \sum_{j} \widetilde{\varepsilon}\left\|y_{j}^{\prime}\right\|_{n+2}^{*}\left\|\varrho_{n+1}^{n}\left(f_{j}\right)\right\|_{n} \\
& \stackrel{(4)}{\leq} \sup _{j}\left\|y_{j}^{\prime}\right\|_{n+2}^{*} \gamma_{j}^{(n)} \stackrel{(8)}{\leq} 1 .
\end{aligned}
$$

Finally, with $S:=L(k) \widetilde{S}$ and $A=x \otimes y$ we get the identity

$$
\begin{aligned}
S \operatorname{id}_{E} \widetilde{\otimes}_{\pi} \varrho_{k}^{n}(B) & +\varepsilon C \\
= & \operatorname{id}_{E} \widetilde{\otimes}_{\pi} \varrho_{k}^{n}\left(\sum_{j} a_{j}^{-1} B_{j} \otimes \varrho_{k+1}^{k}\left(e_{j}\right)\right)+\sum_{j} C_{j} \otimes \varrho_{n+1}^{n}\left(f_{j}\right) \\
= & \sum_{j} a_{j}^{-1} B_{j} \otimes \varrho_{n+1}^{n}\left(\varrho_{k+1}^{n+1}\left(e_{j}\right)\right)+\sum_{j} C_{j} \otimes \varrho_{n+1}^{n}\left(f_{j}\right)
\end{aligned}
$$




$$
\begin{aligned}
& =\sum_{j} B_{j} \otimes \varrho_{n+1}^{n}\left(f_{j}\right)+\sum_{j} C_{j} \otimes \varrho_{n+1}^{n}\left(f_{j}\right) \\
& =\sum_{j} A_{j} \otimes \varrho_{n+1}^{n}\left(f_{j}\right)=\sum_{j}\left(\left\langle\varrho_{m}^{n+1}(y), f_{j}\right\rangle_{j+1} x\right) \otimes \varrho_{n+1}^{n}\left(f_{j}\right) \\
& =x \otimes \varrho_{n+1}^{n}\left(\sum_{j}\left\langle\varrho_{m}^{n+1}(y), f_{j}\right\rangle_{j+1} f_{j}\right)=\operatorname{id}_{E} \widetilde{\otimes}_{\pi} \varrho_{m}^{n}(A),
\end{aligned}
$$

which implies $\left(S_{3}^{\otimes}\right)_{0}$.

Let us summarize our results:

THEOREM 5.9. Let $E$ be a locally convex space, $F$ a nuclear Fréchet space and $\alpha$ a tensor norm. Then

(i) $\operatorname{Tor}_{\alpha}^{l}(E, F)=\operatorname{Tor}_{\pi}^{l}(E, F)$ for all $l \geq 1$.

(ii) $\operatorname{Tor}_{\alpha}^{0}(E, F)=E \widetilde{\otimes}_{\alpha} F$ for $\alpha=\pi$ and $\alpha=\varepsilon$.

(iii) If $\operatorname{Tor}_{\alpha}^{1}(E, F)=0$ then for all exact nuclear $\mathcal{F} \mathcal{S}$-sequences $0 \rightarrow$ $F \rightarrow G \rightarrow H \rightarrow 0$ the $E \widetilde{\otimes}_{\alpha} \cdot$-tensored sequence $0 \rightarrow E \widetilde{\otimes}_{\alpha} F \rightarrow$ $E \widetilde{\otimes}_{\alpha} G \rightarrow E \widetilde{\otimes}_{\alpha} H \rightarrow 0$ is (topologically) exact.

(iv) $\operatorname{Tor}_{\alpha}^{l}(E, F)=0$ for $l \geq 2$.

(v) If $E$ is a proper, complete $(L B)$-space and $\left(F_{n}, \varrho_{m}^{n}\right)$ a fundamental system of Banach spaces for the nuclear Fréchet space $F$, then $\operatorname{Tor}_{\alpha}^{1}(E, F)=0$ if and only if the condition $\left(S_{3}^{K \prime}\right)_{0}$ holds.

Proof. (i) follows from Proposition 5.5(iv).

(ii) Let $\left(F_{n}, \varrho_{m}^{n}\right)$ be a fundamental system of injective Banach spaces. From $\operatorname{Tor}_{\alpha}^{l}\left(E, \prod_{n} F_{n}\right)=\prod_{n} \operatorname{Tor}_{\alpha}^{l}\left(E, F_{n}\right)=0$ for $l \geq 1$ it follows that the canonical resolution

$$
0 \rightarrow F \rightarrow \prod_{n} F_{n} \rightarrow \prod_{n} F_{n} \rightarrow 0
$$

of $F$ is $E \widetilde{\otimes}_{\alpha} \cdot$-acyclic. As $F$ is nuclear, this sequence is a $\otimes$-sequence. Therefore we get (ii) from Corollary 1.7(a).

(iii) By nuclearity we may assume that $\alpha=\pi$. As $F$ is nuclear, the sequence considered is again a $\otimes$-sequence. By Corollary 1.7 (a) we have to prove surjectivity of the map $E \widetilde{\otimes}_{\pi} G \rightarrow E \widetilde{\otimes}_{\pi} H$, which follows immediately from the long exact sequence and (ii).

(iv) is a consequence of Corollary 2.13 and as $F$ is locally injective, (v) follows from Propositions 5.7 and 5.5.

The Tor-theory developed here, when applied to the last standard case, gives new insight into some historical results. In [5, II, $\S 4$, no. 3 , Theorem 1$]$ A. Grothendieck gave a comprehensive characterization of topological properties (to be more precise, of the quasi-barrelledness) of the (complete) projective tensor product of a (DF)-space with a Fréchet space in the case of Köthe coechelon spaces $k^{1}(A)$ and echelon spaces $\lambda^{1}(B)$. In [24] the author generalizes 
this result to arbitrary Fréchet spaces in place of Köthe spaces $\lambda^{1}(B)$, adding homological conditions. In the same paper a corresponding theorem for the injective tensor product is also given. As the proof in [24] shows, we get:

THEOREM 5.10. Let $E$ be either a coechelon space $k^{1}(A)$ of type one and $\alpha=\pi$, or a complete coechelon space $k^{0}(A)$ of type zero and $\alpha=\varepsilon$, and let $F$ be a Fréchet space. Then:

(i) $\operatorname{Tor}_{\alpha}^{0}(E, F)=E \widetilde{\otimes}_{\alpha} F$.

(ii) $\operatorname{Tor}_{\alpha}^{1}(E, F)=0$ if and only if for all short exact $\mathcal{F S}$-sequences the $E \widetilde{\otimes}_{\alpha} \cdot$-tensored sequence is (topologically) exact.

(iii) $\operatorname{Tor}_{\alpha}^{l}(E, F)=0$ for $l \geq 2$.

(iv) If $E$ is proper and $\left(F_{n}, \varrho_{m}^{n}\right)$ a fundamental system of Banach spaces for the Fréchet space $F$ then $\operatorname{Tor}_{\alpha}^{1}(E, F)=0$ if and only if the condition $\left(S_{3}^{K \prime}\right)_{0}$ holds.

At this point we want to show that in acyclic situations the property $\operatorname{Tor}_{\alpha}^{1}(E, G)=0$ passes to quotients of $G$ :

REMARK 5.11. Let $\alpha$ be a tensor norm, $E$ a locally convex space and $G$ a Fréchet space. Suppose further that $\operatorname{Tor}_{\alpha}^{1}(E, G)=0$. In the cases below we also have $\operatorname{Tor}_{\alpha}^{1}(E, H)=0$ for every quotient $H$ of $G$ :

(a) $E$ is a nuclear (DF)-space;

(b) $F:=\operatorname{ker}(G \rightarrow H)$ is locally $E \widetilde{\otimes}_{\alpha}$-acyclic, for instance $G$ nuclear;

(c) $E=k^{1}(A)$ and $\alpha=\pi$;

(d) $E=k^{0}(A)$ complete and $\alpha=\varepsilon$.

Proof. We have shown that in all cases we have $\operatorname{Tor}_{\alpha}^{l}(E, F)=0$ for $l \geq 2$. In cases (a), (c) and (d) even every Banach space is $E \widetilde{\otimes}_{\alpha}$-acyclic. Applying the long exact sequence to $0 \rightarrow F \rightarrow G \rightarrow H \rightarrow 0$ gives the exact $\mathcal{L S}$-sequence $\cdots \rightarrow \operatorname{Tor}_{\alpha}^{1}(E, G) \rightarrow \operatorname{Tor}_{\alpha}^{1}(E, H) \rightarrow \operatorname{Tor}_{\alpha}^{2}(E, F) \rightarrow \cdots$ and therefore $\operatorname{Tor}_{\alpha}^{1}(E, H)=0$.

Theoretical applications of the Tor-theory established here such as the characterization of quojections or the property $(\Omega)$ of D. Vogt and M. J. Wagner can be found in [24], while practical applications such as the solutions of (DF)-valued partial differential operators with constant coefficients will be treated in [25], where we shall use a method of H. J. Petzsche ([22]) for decomposing elementary tensors.

\section{References}

[1] R. Braun and D. Vogt, A sufficient condition for $\operatorname{proj}^{1} \mathcal{X}=0$, Michigan Math. J. 44 (1997), 149-156.

[2] A. Defant and K. Floret, Tensor Norms and Operator Ideals, North-Holland Math. Stud. 176, North-Holland, 1993. 
[3] A. Defant and K. Floret, Topological tensor products and the approximation property of locally convex spaces, Bull. Soc. Roy. Sci. Liège 58 (1989), 29-51.

[4] L. Frerick and J. Wengenroth, A sufficient condition for vanishing of the derived projective limit functor, Arch. Math. (Basel) 67 (1996), 296-301.

[5] A. Grothendieck, Produits tensoriels topologiques et espaces nucléaires, Mem. Amer. Math. Soc. 16 (1955); Erratum, Ann. Inst. Fourier (Grenoble) 6 (1955/56), 117-120.

[6] J. Harksen, Tensornormtopologien. Dissertation, Kiel, 1979.

[7] R. Hollstein, Inductive limits and $\varepsilon$-tensor products, J. Reine Angew. Math. 319 (1980), 38-62.

[8] - Extension and lifting of continuous linear mappings in locally convex spaces, Math. Nachr. 108 (1982), 275-297.

[9] - Tensor sequences and inductive limits with local partition of unity, Manuscripta Math. 52 (1985), 227-249.

[10] H. Jarchow, Locally Convex Spaces, Stuttgart, B. G. Teubner, 1981.

[11] W. Kaballo, Lifting-Sätze für Vektorfunktionen und ( $\varepsilon L)$-Räume, J. Reine Angew. Math. 309 (1979), 55-85.

[12] W. Kaballo und D. Vogt, Lifting-Probleme für Vektorfunktionen und $\otimes$-Sequenzen, Manuscripta Math. 32 (1980), 1-27.

[13] M. Langenbruch, Characterization of surjective partial differential operators on spaces of real analytic functions, Studia Math. 162 (2004), 53-96.

[14] J. Lindenstrauss and L. Tzafriri, Classical Banach Spaces, Lecture Notes in Math. 338, Springer, Berlin, 1973.

[15] E. M. Mangino, Complete projective tensor product of (LB)-spaces, Arch. Math. (Basel) 64 (1995), 33-41.

[16] —, (LF)-spaces and tensor products, Math. Nachr. 185 (1997), 149-162.

[17] R. Meise and D. Vogt, Introduction to Functional Analysis, transl. from the German by M. S. Ramanujan, Oxford Grad. Texts in Math. 2, Clarendon Press, Oxford, 1997.

[18] S. N. Melikhov, Absolutely convergent series in the canonical inductive limits, Mat. Zametki 39 (1986), 877-886 (in Russian); English transl.: Math. Notes 39 (1986), 475-480.

[19] V. P. Palamodov, Homological methods in the theory of locally convex spaces, Uspekhi Mat. Nauk 26 (1971), no. 1, 3-65 (in Russian); English transl.: Russian Math. Surveys 26 (1971), no. 1, 1-64.

[20] P. Pérez Carreras and J. Bonet, Barrelled Locally Convex Spaces, North-Holland Math. Stud. 131, North-Holland, Amsterdam, 1987.

[21] A. Peris, Quasinormable spaces and the problem of topologies of Grothendieck, Ann. Acad. Sci. Fenn. Ser. A I Math. 19 (1994), 167-203.

[22] H.-J. Petzsche, Some results of Mittag-Leffler-type for vector valued functions and spaces of class $\mathcal{A}$, in: Functional Analysis: Surveys and Recent Results II (Paderborn, 1979), North-Holland Math. Stud. 38, North-Holland, Amsterdam, 1980, 183204.

[23] J. J. Rotman, An Introduction to Homological Algebra, Pure Appl. Math. 85, Academic Press, New York, 1979.

[24] O. Varol, A generalization of a theorem of A. Grothendieck, Math. Nachr. 280 (2007), 313-325.

[25] —, A decomposition lemma for elementary tensors, Arch. Math. (Basel), to appear.

[26] D. Vogt, On the functors $\operatorname{Ext}^{1}(E, F)$ for Fréchet spaces, Studia Math. 85 (1987), 164-197. 
[27] D. Vogt, Topics on projective spectra of (LB)-spaces, in: Advances in the Theory of Fréchet Spaces (Istanbul, 1988), NATO Adv. Sci. Inst. Ser. C 287, Kluwer, 1989, $11-27$.

[28] - Some results on continuous linear maps between Fréchet spaces, in: Functional Analysis: Surveys and Recent Results III (Paderborn, 1983), North-Holland Math. Stud. 90, North-Holland, Amsterdam, 1984, 349-381.

[29] - On the solvability of $P(D) f=g$ for vector valued functions, preprint, 1983.

[30] C. A. Weibel, An Introduction to Homological Algebra, Cambridge Stud. Adv. Math. 38, Cambridge Univ. Press, Cambridge, 1994.

[31] J. Wengenroth, Derived Functors in Functional Analysis, Lecture Notes in Math. 1810, Springer, Berlin, 2003.

Fachbereich C - Mathematik

Bergische Universität Wuppertal

D-42097 Wuppertal, Germany

E-mail: varol@math.uni-wuppertal.de

Received November 7, 2005

Revised version January 16, 2007 